\title{
Impacto de los Programas de Transferencias de Ingresos en la Calidad de la Vivienda Familiar: Evidencia para el Plan de Inclusión Social, Argentina
}

Alfredo Palacios

María Laura Alzúa

19 de Diciembre de 2016 


\title{
Impacto de los Programas de Transferencias de Ingresos en la Calidad de la Vivienda Familiar: Evidencia para el Plan de Inclusión Social, Argentina*
}

\author{
Alfredo Palacios \\ Universidad Nacional de La Plata (UNLP)
}

\begin{abstract}
Resumen
Se busca identificar si el Plan de Inclusión Social, un programa de transferencias de ingresos implementado en San Luis en 2003, tuvo efecto sobre el porcentaje de individuos que habitan viviendas de calidad inadecuada. Se construye un panel de datos a nivel aglomerados para el periodo 1996-2006, y se utiliza el Método del Control Sintético para identificar el impacto causal del programa sobre la variable de interés. Los resultados sugieren que el programa posibilitó una reducción de 1.85 puntos porcentuales en el porcentaje de individuos que habitan viviendas de calidad inadecuada, siendo este resultado robusto a diferentes pruebas de falsación.
\end{abstract}

Palabras clave: Transferencia de ingresos, Vivienda, Condiciones habitacionales, Bienestar, Método del Control Sintético.

Códigos JEL: O12, I38, D10.

\footnotetext{
* El presente trabajo constituye mi tesis de Maestría en Economía, requerida por el Departamento de Economía de la Universidad Nacional de La Plata. Estuvo dirigida por María Laura Alzúa, y contó con apoyo del proyecto "Mejorando Capacidades en Evaluación de Impacto en América Latina" de IDRC, Canadá y CEDLAS-UNLP. Se agradecen los comentarios de Guillermo Cruces y Mariano Rabassa, integrantes del jurado de tesis, de Julia Gabosi, Ignacio Carballo y de los participantes del Seminario de Avances de Tesis de la Maestría en Economía - UNLP y de la LI Reunión Anual de la Asociación Argentina de Economía Política (AAEP). Los errores u omisiones son responsabilidad del autor. Correspondencia a: alfredopalacios77@gmail.com.
} 


\section{Introducción}

Los programas de transferencias de ingresos han sido una opción de política social ampliamente utilizada, y estudiada, durante las últimas décadas en los países de la región. Ejemplos de ello lo constituyen los programas Oportunidades en México, Red Solidaria en El Salvador, Familias en Acción en Colombia, entre muchos otros. Si bien parece existir un consenso acerca de que estos programas han tenido efectos beneficiosos sobre la población objetivo, al menos en términos de las condicionalidades que requieren para su percepción (Fiszbein et al., 2009; Rawlings and Rubio, 2005; Handa and Davis, 2006), existen ciertos elementos o aspectos de dichos programas que ameritan un análisis particular.

Una cuestión de especial relevancia, al menos desde la perspectiva de política económica y social, es si estos programas de transferencias de ingresos podrían tener efectos indirectos (es decir, no vinculados a condicionalidades) sobre los hogares beneficiarios, con implicancias en su bienestar de mediano y largo plazo (Banerjee and Mullainathan, 2010; Barrientos, 2012; Evans and Popova, 2016). En este sentido, la literatura sobre los efectos no intencionados de los programas sociales en la región es por demás limitada y, en general, se encuentra centrada en la identificación de efectos sobre la participación laboral de los beneficiarios y la tasa de fecundidad de las familias, y en menor medida sobre el uso que hace el hogar de los montos transferidos. Dentro de este último sub-grupo de literatura, los estudios se han focalizado en la identificación de efectos de bienestar de corto plazo, como consumo de alimentos e inversión en activos productivos básicos en un contexto rural.

Bajo este marco, el presente trabajo tiene por objetivo general contribuir a este último sub-grupo de literatura mediante el análisis de un posible efecto indirecto que un programa de transferencias de ingresos podría tener sobre la población beneficiaria en un contexto urbano, considerando una variable de resultado que posea implicancias en el bienestar de mediano y largo plazo de los hogares. En particular, se propone identificar si el programa de transferencias de ingresos denominado "Plan de Inclusión Social", implementado en la provincia de San Luis, Argentina, en mayo de 2003, podría haber llevado a los hogares a mejorar la calidad de sus viviendas, posibilitando así una reducción en el porcentaje de individuos que habitan viviendas familiares de calidad inadecuada a nivel provincial. De acuerdo a mi mejor conocimiento, este trabajo constituye la primera evaluación de impacto de un programa de transferencias de ingresos en términos de la mejora de la calidad de la vivienda familiar.

El Plan de Inclusión Social surge en mayo de 2003 en la provincia de San Luis como una respuesta local al contexto de crisis económica y social de Argentina de 2001-2002. Se trató de un programa de transferencias de ingresos a la población desocupada al estilo del Plan Nacional 
Jefes y Jefas de Hogar Desocupados (PJJHD), aunque con algunas características superadoras, como un mayor monto en concepto de transferencia por beneficiario, la actualización de la misma con frecuencia anual, y la no existencia de restricciones en el número de miembros del hogar que podían percibir tal transferencia. Estas características salientes del Plan de Inclusión Social podrían haber dado lugar a una mejora sustancial en el ingreso de los hogares beneficiarios, el que podría haber sido utilizado, al menos parcialmente, para mejorar la calidad de la vivienda familiar.

A partir de aquí, el trabajo estará organizado de la siguiente manera. La sección 2 presenta los antecedentes de investigación en la región sobre los efectos indirectos de los programas de transferencias de ingresos con foco en la región. La sección 3 brinda una descripción del Plan de Inclusión Social, incluyendo una revisión de los principales trabajos que lo analizaron. El marco teórico que da sustento a este trabajo es expuesto en la sección 4, en donde se plantea un modelo de ciclo de vida con elección de la calidad de la vivienda familiar por parte del hogar en situación de pobreza. La sección 5 presenta la metodología de evaluación de impacto a ser utilizada, junto con el modelo teórico que la sustenta, su implementación computacional y sus alcances y limitaciones. La sección 6 describe la base de datos utilizada en el presente estudio, su proceso de construcción y fuentes de información. Los resultados obtenidos son analizados en la sección 7 , donde se incluye además una serie de pruebas de robustez, mientras que la sección 8 presenta los resultados que surgen al explotar, a modo de experimento natural, el plebiscito al que fue sometido el programa en agosto de 2004. Finalmente, la sección 9 expone las conclusiones del trabajo.

\section{Antecedentes}

La literatura sobre los efectos no intencionados de los programas de transferencias de ingresos en la región es relativamente limitada y, en general, se encuentra centrada en aspectos vinculados con los incentivos a la participación laboral de los beneficiarios, la tasa de fecundidad de las familias y, en menor medida, el uso que hacen los hogares de los montos transferidos.

A continuación, se realizará una breve revisión de la evidencia en términos de la participación laboral y fecundidad de las familias, seleccionando para ello estudios que cuenten con validez interna (análisis de programas con diseño experimental) y externa (análisis comparativo de programas implementados en más de un país), para luego centrarnos en la revisión de la literatura sobre los efectos no intencionados de los programas sociales en términos del uso de las trasferencias por parte de los hogares.

Los trabajos que analizan los efectos de los programas sociales sobre variables de participa- 
ción laboral es numerosa y, en general, presenta resultados consistentes. Por caso, Alzúa et al. (2013) examina tres programas de transferencias de ingresos con diseño experimental implementados en México (Progresa), Nicaragua (Red de Protección Social) y Honduras (Programa de Asignación Familiar), obteniendo por resultado general que ninguno de estos programas llevó a cambios estadísticamente significativos en las variables de oferta laboral de los hogares beneficiarios. En similar dirección, Banerjee et al. (2015) amplía el estudio previo al considerar más programas en más países, especificaciones alternativas, etc., y reafirma los resultados obtenidos.

En relación a los posibles efectos de este tipo de programas sobre variables de fecundidad de los hogares, la literatura es relativamente limitada, aunque parece haber cierta consistencia en los resultados obtenidos. En particular, Stecklov et al. (2007) analiza bases de micro-datos de tres programas de transferencias de ingresos que cuentan con diseño experimental en Honduras (Programa de Asistencia Familiar), México (Progresa) y Nicaragua (Red de Protección Social), y concluye que, salvo en el caso de Honduras, no hay evidencia que sugiera que estos programas sociales incrementen la fecundidad de los hogares beneficiarios. En la misma línea, Glassman et al. (2013) realiza una revisión sistemática de estudios que estimaron el efecto de programas sociales en países de la región sobre variables de salud de madres y niños en general, y de fecundidad en particular, y expresan conclusiones similares a las del estudio anterior.

Como fuera sugerido previamente, la literatura sobre los efectos no intencionados de los programas de transferencias de ingresos en términos de su uso por parte de las familias beneficiarias es muy limitada y, en general, se encuentra centrada en la identificación de efectos de corto plazo, como consumo de alimentos e inversión en activos productivos básicos bajo un contexto rural.

Por caso, Dasso and Fernández (2013) analiza si los beneficiarios del programa de transferencias condicionadas Juntos de Perú modifican su patrón de consumo de alimentos según el momento de tiempo en el cual reciben la transferencia. Para ello, explotan las diferencias que existen entre las fechas de entrevistas y fechas de pago generando, de manera exógena, que algunos beneficiarios sean entrevistados a días de haber recibido la transferencia y otros no. Los autores identifican un incremento de entre 10 y $20 \%$ en el gasto en alimentos en el periodo de pago, y tal incremento parece ser explicado por un mayor consumo de alimentos que son costosos y poco nutritivos (gaseosas, chocolates, etc.). Un estudio similar, pero que presenta resultados opuestos, es el realizado por Angelucci and Attanasio (2013), quienes estiman funciones de demanda de alimentos en general y de aquellos que poseen un alto contenido de proteínas (carne, pescado, etc.) en hogares beneficiarios y no beneficiarios de Progresa en México. A través de la utilización de modelos estructurales, los autores identifican que los hogares que se encuentran 
en áreas donde se implementó el programa experimentaron un incremento en su demanda de alimentos, con una mayor participación de aquellos que poseen un alto contenido proteico.

Por otra parte, Angelucci and De Giorgi (2009) avanzan metodológicamente en la identificación de cómo las transferencias de ingresos a hogares elegibles de Progresa en México afectan de manera indirecta el consumo de los hogares no elegibles que habitan las mismas áreas. A través del análisis de datos experimentales, los autores identifican que el consumo de alimentos de los hogares no elegibles que habitan las mismas áreas que los elegibles se incrementa en aproximadamente un $10 \%$ (cerca de la mitad del efecto observado en los hogares elegibles). Tal incremento en el consumo de alimentos de los hogares no elegibles es explicado por préstamos de familiares y amigos que son receptores de las transferencias, lo que a su vez lleva a una reducción de los ahorros precautorios por parte de las familias no beneficiarias.

Un estudio de particular interés es el realizado por Gertler et al. (2012), quien utiliza información de Progresa para hallar que los hogares rurales que reciben el programa emplean parte de las transferencias para realizar inversiones en activos productivos agrícolas (animales, tierra, etc.), incrementando su ingreso por tal actividad en aproximadamente un $10 \%$ luego de 18 meses de recibir el programa. Se identifica además que, después de 4 años de que los hogares de control fueran incorporados al programa, las acciones de inversión posibilitaron que el consumo en los hogares originalmente de tratamiento sea un 5.6\% mayor en relación a los hogares inicialmente de control, realizando así un importante aporte al debate sobre si los programas de transferencias de ingresos podrían incrementar el estándar de vida de los hogares beneficiarios desde una perspectiva intertemporal. En similar dirección, aunque con resultados opuestos, Maluccio (2010) examina el efecto del programa de transferencias de ingresos Red de Protección Social de Nicaragua sobre un conjunto de indicadores de consumo e inversiones productivas en hogares rurales. Haciendo uso de un panel de datos que surge de una intervención aleatorizada del programa, el autor identifica un claro incremento en el gasto en alimentos en los hogares tratados durante el periodo de vigencia del programa, desapareciendo tal efecto con el cese de la intervención. Adicionalmente, la evidencia de que el programa haya tenido un efecto positivo en la inversión en activos productivos por parte de los hogares beneficiarios resulta sumamente limitada.

Bajo este marco, el trabajo tiene por objetivo contribuir a esta literatura mediante el análisis de un posible efecto indirecto que un programa de transferencias de ingresos podría tener sobre los hogares en un contexto urbano, considerando una variable de resultado posea implicancias en el bienestar de mediano y largo plazo de la población. En particular, se propone identificar si el programa de transferencias de ingresos Plan de Inclusión Social podría haber llevado a 
los hogares beneficiarios a mejorar la calidad de sus viviendas, resultando en una reducción del porcentaje de individuos que habitan viviendas de calidad inadecuada a nivel provincial ${ }^{1}$.

\section{Descripción del Programa "Plan de Inclusión Social"}

Bajo el contexto de crisis económica y social experimentado a nivel nacional durante los años 2001 y 2002, la provincia de San Luis decide implementar, en mayo de 2003, un programa de transferencias de ingresos denominado Plan de Inclusión Social "Trabajo por San Luis", mediante la Ley provincial 5373. Al igual que otros programas de transferencias de ingresos, el Plan de Inclusión Social tuvo por objetivo general reducir la situación de pobreza en la que se encontraba la población, exigiendo como contraprestación o condicionalidad la realización de ciertas actividades laborales básicas. Esta clase de condicionalidad permitiría alcanzar un objetivo de política complementario, representado por la "inclusión social" de los beneficiarios a través de la cultura del trabajo (Suárez Godoy, 2004).

En la práctica, se trató de un programa de transferencias de ingresos a la población desocupada al estilo del Plan Nacional Jefes y Jefas de Hogar Desocupados (PJJHD)², aunque el programa provincial presentó algunas características superadoras en relación a su par nacional. En primer lugar, el monto en concepto de transferencia por beneficiario del Plan de Inclusión Social en su primer año de implementación fue de $\$ 300$, claramente superior al de PJJHD, de $\$ 150$. Segundo, la transferencia monetaria del programa provincial fue actualizada con frecuencia anual, conservando buena parte de su poder de compra en el tiempo, mientras que en el caso del Plan Jefes y Jefas tal actualización ha sido por demás limitada, al menos en relación al salario mínimo de la economía (Golbert, 2007). En tercer lugar, en el Plan de Inclusión Social -a diferencia del PJJHD- no existían restricciones en el número de miembros del hogar que podían percibir tal transferencia, siempre que cumplieran con los requerimientos básicos del programa, por demás flexibles (Suárez Godoy, 2004; Olguín et al., 2012). Estas características salientes del Plan de Inclusión Social podrían haber dado lugar a una mejora sustancial en el ingreso de los hogares beneficiarios, el que podría haber sido utilizado -al menos parcialmente- para mejorar la calidad de la vivienda que habitan.

\footnotetext{
${ }^{1}$ Existe literatura económica y médica que ha analizado los efectos de la mejora de la calidad de la vivienda sobre variables de salud de adultos y niños, principalmente, incidencia de diarrea, parásitos, anemia, entre otros. Por caso, ver los trabajos de Aiga et al. (1999) y Galiani et al. (2005) sobre la mejora en el suministro y conexión al agua de red, Cattaneo et al. (2009) sobre el impacto de un programa de mejora del material del piso de la vivienda familiar, Wolff et al. (2001), Choudhary et al. (2002) y Galiani et al. (2016) sobre la provisión de viviendas de emergencia en contextos de pobreza extrema, entre otros.

${ }^{2}$ Numerosos trabajos han analizado el Plan Nacional Jefes y Jefas de Hogar Desocupados en términos de su diseño, focalización e impacto sobre el nivel de pobreza y desempleo. Ver Galasso and Ravallion (2004); Golbert (2004); Cruces et al. (2008); Cruces and Gasparini (2008), entre otros.
} 
Es importante mencionar que, según información de la Dirección de Gasto Público y Programas Sociales de 2006 (DGPyPS, 2006), dependiente del Ministerio de Economía de la Nación, el Plan de Inclusión Social ha sido uno de los programas sociales provinciales más importantes del país, contando con más de 44 mil beneficiarios (superado sólo por el Programa "Barrios Bonaerenses", de la Provincia de Buenos Aires, con casi 65 mil), con uno de los montos de transferencia por beneficiario más elevados a nivel nacional, de $\$ 390$ (sólo superado por el Programa "Entrenamiento Laboral" de Tierra del Fuego, con una transferencia de \$500), siendo así el programa social provincial de mayor gasto público anual, de 207 millones de pesos ejecutados, claramente superior al gasto anual del Programa "Barrios Bonaerenses", de 119 millones ${ }^{3}$.

Desde su inicio el programa no contó con un criterio demarcado de inclusión o exclusión de potenciales beneficiarios, en tanto el Artículo 3 de la Ley 5373 establece que "(El Plan de Inclusión Social) está dirigido a todos los ciudadanos de San Luis desocupados, dispuestos a mejorar sus posibilidades de conseguir empleo mediante la inclusión de la cultura del trabajo. Se garantiza el acceso al Plan de las madres solteras, mujeres jefas de hogar, mujeres mayores de cuarenta (40), cincuenta (50) y sesenta (60) años, todas las personas con capacidades diferentes, hombres mayores de cuarenta (40) años, todos los jóvenes, mujeres y hombres mayores de dieciocho (18) años y de todo sector de la población en estado de emergencia social". Los interesados en participar del programa debían acreditar su identidad, ser mayores de 18 años, tener dos años de residencia en la provincia (sobre esto se efectuarían excepciones presentando constancia de escolaridad de los hijos) y no contar con otro tipo de beneficio u ocupación.

Olguín and Páez (2005) brindan una caracterización de los ocupados en planes sociales en San Luis a partir de la información provista por la Encuesta Permanente de Hogares (EPH) para el segundo semestre de 2004, e identifican que casi el $42 \%$ de los ocupados en programas sociales en la provincia son jefes de hogar (cifra de casi $53 \%$ para el total de ocupados), aproximadamente un $31 \%$ son cónyugues (casi un $23 \%$ para el total de ocupados), el $60 \%$ de los ocupados en el programa son mujeres (cifra de aproximadamente el $40 \%$ para el total de ocupados), y el $25 \%$ de los beneficiarios poseen entre 15 y 25 años de edad (aproximadamente el $19 \%$ para el total de ocupados).

Para la implementación del programa, el gobierno provincial destinó una asignación presupuestaria de 177 millones de pesos (aproximadamente un 25\% del presupuesto de la provincia), desagregado en 45.000 beneficiarios quienes durante el primer año recibieron una colaboración

\footnotetext{
${ }^{3}$ En términos presupuestarios, los principales "aportantes" para el funcionamiento del Plan de Inclusión Social fueron las partidas tradicionalmente destinadas a obra pública, entre las que se destacan las asignadas a construcción de viviendas, provisión de agua potable y cloacas (Páez et al., 2008). Ello llevó a que el gasto público provincial en programas de vivienda, expresado en términos del gasto público social, pasara de representar más del $20 \%$ promedio para el quinquenio 1998-2002 a sólo un 5.5\% promedio para el periodo 2004-2006, esto es, antes y después de la implementación del Plan de Inclusión Social.
} 
económica de carácter no remunerativo por todo concepto de $\$ 300$ mensuales, y 500 beneficiarios (coordinadores) cuya colaboración económica fue de $\$ 450$, siendo estas cifras actualizadas con frecuencia anual. Páez et al. (2008) identifican que la puesta en marcha del programa implicó un cambio sustancial en la orientación del Gasto Público Social (GPS) de la provincia, el cual pasó de estar centrado en la ejecución de obra pública para dar un lugar preponderante al Plan de Inclusión Social (el cual representó casi el $45 \%$ del GPS en 2004), esto es, la mayor parte del financiamiento destinado al programa surgió de partidas históricamente asignadas a las categorías Obras de infraestructura para la producción, Infraestructura hídrica (obras de saneamiento), Ejecución de obras (arquitectura) y Vialidad provincial (ampliación de la red vial).

En relación a la constraprestación requerida a los beneficiarios, el Artículo 9 de la Ley 5373 establece que "las prácticas a desarrollar por los beneficiarios en el marco de esta normativa tendrán una duración de ocho horas diarias, cinco días a la semana. El Poder Ejecutivo podrá establecer condiciones especiales para los casos de personas con capacidades diferentes debidamente justificados que no puedan cumplir el horario establecido precedentemente", y entre las tareas a desarrollar se encontraron la limpieza y mantenimiento de caminos y canales de riego, la forestación de espacios públicos, entre otras actividades comunitarias básicas.

Un aspecto de especial interés, mencionado anteriormente, es que la percepción del beneficio del Plan de Inclusión Social era incompatible con la que podrían proveer otros programas sociales de carácter nacional vigentes en la provincia, como lo fue en su momento el Plan Jefes y Jefas de Hogar Desocupados (implementado en mayo de 2002) o más recientemente la Asignación Universal por Hijo para la Protección Social (implementada en octubre de 2009). En este sentido, Olguín et al. (2012) afirma que al inicio del Plan de Inclusión Social la mayoría de los beneficiarios del Plan Jefes y Jefas de Hogar optaron por recibir el beneficio del programa provincial, cuyo monto de transferencia en 2003 era de $\$ 300$, el doble de su par nacional, mientras que en el momento de la implementación de la Asignación Universal por Hijo una gran proporción de beneficiarios del Plan de Inclusión habría optado por el programa de protección social nacional.

Además de los estudios ya mencionados, otros artículos han analizado el Plan de Inclusión Social desde el campo de la Economía. En particular, Olguín and Páez (2005) y Olguín et al. (2005) examinan la incidencia del Plan de Inclusión Social en la medición de la desocupación y la pobreza utilizando información provista por la EPH para el año 2004 y obtienen que, considerando como ocupados a quienes se autodefinen como beneficiarios de planes sociales, la tasa de desocupación en San Luis fue del $1.2 \%$, pero cuando se excluye del cálculo a dichos 
beneficiarios la tasa aumenta a un $21.4 \%$, siendo esta medida de incidencia de planes sociales mucho menor en otros aglomerados de la región, como San Juan y Mendoza. De la misma manera, los autores afirman que la tasa de pobreza a nivel de individuos en San Luis fue del $42 \%$ y la de indigencia del $14.2 \%$, pero si estas medidas son recalculadas restando del ingreso el monto percibido en concepto de planes sociales la tasa de pobreza e indigencia aumentarían a $49 \%$ y $28.8 \%$, respectivamente, siendo estas medidas de incidencia mucho menores en los aglomerados vecinos ${ }^{4}$.

\section{Marco teórico}

Partimos de considerar un modelo de ciclo de vida con elección de la calidad de la vivienda familiar por parte del hogar en situación de pobreza ${ }^{5}$. Asumimos que los hogares viven durante $T$ periodos. En el periodo $t<T$ el hogar maximiza su utilidad $U($.$) que depende del consumo$ de vivienda $h_{t}$ y del consumo del resto de los bienes $C_{t}$.

La vivienda familiar $h_{t}$ puede ser de dos tipos según su nivel de calidad, la que se define a partir de poseer una serie de atributos como conexión al agua corriente, baño de uso exclusivo, cantidad de cuartos y calidad de los materiales. Si la vivienda carece de al menos uno de estos atributos diremos que es una vivienda de calidad inadecuada, y la clasificaremos como tipo 1 , mientras que si posee todos los atributos diremos que es una vivienda de calidad adecuada, clasificándola como tipo 2. De acuerdo con ello, el hogar elige la vivienda familiar $h_{t}$, donde $h_{t} \in\{1,2\}$.

La función valor de este problema en su forma recursiva es

$$
V_{t}\left(M_{t}, p_{t}, h_{t-1}\right)=\operatorname{Max} U\left(C_{t}, h_{t}\right)+\beta E_{t} V_{t+1}\left(M_{t+1}, p_{t+1}, h_{t}\right)
$$

sujeto a

$$
\begin{cases}M_{t}-C_{t}-\kappa p_{t} I\left(h_{t}=1\right) & \text { si } h_{t}=1 \\ M_{t}-C_{t}-p_{t} I\left(h_{t}=2\right) & \text { si } h_{t}=2\end{cases}
$$

donde $M$ es el ingreso del hogar en el periodo $t, p$ es el precio de la vivienda de calidad adecuada y $\kappa$ es una fracción que representa que el precio de la vivienda de calidad inadecuada

\footnotetext{
${ }^{4}$ Existe una serie de trabajos que han examinado el programa desde el enfoque de las Ciencias Políticas, la Sociología y la Psicología. Entre ellos se destacan las investigaciones de Dorzán and Arias (2005) y Vilchez (2013), quienes indagan sobre el discurso oficial del programa y la normativa jurídica correspondiente, y los de Vilchez and Feigelman (2009), Bussetti (2009) y Feigelman (2008), quienes discuten los conceptos de ciudadanía, inclusión social y vulnerabilidad a la luz de la experiencia del Plan de Inclusión Social.

${ }^{5}$ El modelo presentado en esta sección se encuentra inspirado en los trabajos de Attanasio et al. (2012), Barrientos (2012) y Jacob et al. (2014).
} 
es inferior al de la vivienda de calidad adecuada ${ }^{6}$.

La función de utilidad del hogar $U($.$) asume la forma de una función con aversión al riesgo$ relativo constante del tipo

$$
U\left(C_{t}, h_{t}\right)=\frac{C_{t}^{1-\gamma}}{1-\gamma} \exp \left(\phi\left(h_{t}\right)\right) \begin{cases}0<\phi<1 & \text { si } h_{t}=1 \\ \phi=1 & \text { si } h_{t}=2\end{cases}
$$

donde $\phi$ es un parámetro que representa la utilidad relativa de tener una vivienda familiar de mayor calidad. Por otra parte, la restricción presupuestaria del hogar es igual a

$$
M_{t}=w_{t}+A_{t}
$$

donde $w_{t}$ es el ingreso laboral del hogar en el periodo $t$, el cual se asume sigue un proceso random walk, y $A_{t}$ representa el ingreso proveniente de transferencias del gobierno en el periodo $t$, esto es,

$$
\begin{gathered}
w_{t}=b_{t}+\xi_{t}, \text { donde } \xi \sim N\left(\frac{-\sigma_{\xi}^{2}}{2}, \sigma_{\xi}^{2}\right) \\
\begin{cases}A_{t}>0 & \text { si } t \geq t_{0} \\
A_{t}=0 & \text { si } t<t_{0}\end{cases}
\end{gathered}
$$

donde $t_{0}$ es el año de implementación del programa de transferencias de ingresos ${ }^{7}$.

Se postula que el programa podría haber beneficiado a los hogares mediante la expansión de su restricción presupuestaria y la reducción de la incertidumbre sobre ingresos presentes y futuros, lo que a su vez, podría haber llevado al hogar a incrementar su demanda de $h_{t}$ de tipo 1 a tipo 2 .

Esta hipótesis es representada en la Figura 1, en donde $E$ es el nivel de equilibrio de consumo de $C_{t}$ y $h_{t}$ del hogar antes de la implementación del programa de transferencias de ingresos. Una vez implementado el mismo, la restricción presupuestaria $M$ se expande en una magnitud $A$ hacia $M^{\prime}$, ampliando el espacio de posibilidades de consumo de $C$ y $h$.

\section{[FIGURA 1]}

\footnotetext{
${ }^{6}$ En este modelo asumimos que el hogar en situación de pobreza no tiene acceso a mercados de crédito (Barrientos, 2012).

${ }^{7}$ Notar que el modelo aquí presentado parte de considerar que el hogar toma la decisión de participar en el programa de transferencias de ingresos. Tal decisión está lejos de ser trivial, habida cuenta de que en ella podrían entrar en juego factores vinculados con la participación de los miembros del hogar en el mercado laboral formal/informal, el estigma social que podrían sufrir derivado de participar en el programa, entre otros. Para una discusión sobre estos factores ver el trabajo seminal de Ashenfelter (1983).
} 
Resulta claro que el nuevo punto de equilibrio podría determinarse en un punto como $E^{\prime}$, donde no cambia el nivel de $h_{t}$ demandado por el hogar (y se incrementa el consumo del resto de los bienes $C_{t}$ ), o en un punto como $E^{\prime \prime}$, donde el hogar incrementaría el nivel de $h_{t}$, pasando de tipo 1 a tipo 2, siendo esta última situación nuestra hipótesis de trabajo.

Desde el punto de vista aplicado, y a partir de la disponibilidad de información, el modelo de comportamiento del hogar representativo descripto anteriormente puede ser agregado a nivel de la provincia donde el programa fue implementado (Attanasio et al., 2012; Mas-Colell et al., 1995).

\section{Metodología de estimación}

Como fuera mencionado anteriormente, el objetivo de este trabajo será identificar el posible efecto del programa de transferencias de ingresos Plan de Inclusión Social sobre el porcentaje de individuos que habitan viviendas cuya calidad sea considerada inadecuada bajo un contexto urbano. Para ello, se define un indicador dicotómico de calidad de la vivienda a partir de cuatro aspectos de interés, que se encuentran presentes en la Encuesta Permanente de Hogares (EPH): a) Falta de instalación de agua corriente en la vivienda, b) Ausencia de baño de uso exclusivo, c) Situación de hacinamiento (cuatro o más personas por cuarto), y d) Vivienda construida con materiales de mala calidad. Estas medidas permitirán identificar, bajo el enfoque de la unión, si un individuo habita una vivienda de calidad inadecuada o no, y colapsando este indicador a nivel de aglomerado se obtiene el porcentaje de individuos que habitan viviendas familiares de calidad inadecuada para tal unidad de análisis. Esta definición se encuentra enmarcada dentro las consideraciones de ONU-Hábitat (2010), y es similar a la utilizada por Conconi (2011) para caracterizar la dimensión vivienda en su trabajo sobre indicadores de pobreza multidimensional en Argentina.

Debido a que el programa a evaluar fue implementado en sólo una provincia argentina, permaneciendo las restantes sin una intervención de similares características, se propone como metodología de evaluación al Método del Control Sintético (MCS), desarrollado por Abadie and Gardeazabal $(2003)^{8}$. En líneas generales, el MCS implica la construcción de una unidad

\footnotetext{
${ }^{8}$ Es importante mencionar que, durante el periodo de análisis propuesto, existieron otros programas sociales en otras provincias, lo que podría poner en duda el supuesto de que las potenciales unidades de control permanecieron sin una intervención similar. Sin embargo, y según información provista por la Dirección de Gasto Público y Programas Sociales (DGPyPS, 2006), la incidencia del Plan de Inclusión Social en términos de población beneficiaria fue considerablemente superior a lo evidenciado por el resto de los programas sociales provinciales: mientras que el Plan de Inclusión Social tuvo 16.28 beneficiarios cada 100 habitantes mayores de 18 años, en Neuquén tal medida fue de sólo 4.97, y en La Pampa de 2.3, siendo éstas las tres provincias con mayor incidencia de programas sociales locales. El promedio nacional de incidencia de programas sociales provinciales para este periodo fue de 0.77. En cualquier caso, las provincias de Neuquén y La Pampa no serán consideradas en el panel de donantes para la construcción del control sintético.
} 
de control sintética como una combinación convexa de múltiples unidades de control. Los ponderadores que definen la unidad de control sintética son elegidos de manera tal que sea el mejor ajuste a las características relevantes de la unidad tratada durante el periodo pre-tratamiento, y los resultados post-tratamiento de la unidad de control sintética son luego utilizados para estimar los resultados que deberían haber sido observados en la unidad tratada en ausencia de intervención ${ }^{9}$.

\subsection{El Modelo}

Partimos de considerar que observamos $J+1$ unidades o provincias. Sin pérdida de generalidad, podemos asumir que sólamente la primera provincia es expuesta a la intervención de interés, de manera que habrá $J$ provincias restantes que podrían actuar como potenciales controles. Adicionalmente, puede asumirse que la provincia que recibió la intervención continúa en estado de tratamiento luego del periodo que da inicio al mismo.

Sea $Y_{i t}^{N}$ el resultado que debería ser observado para la provincia $i$ en el periodo $t$ en ausencia de intervención, para las unidades $i=1, \ldots, J+1$, y los periodos de tiempo $t=1, \ldots, T$. Sea $T_{0}$ el número de periodos pre-intervención, con $1 \leq T_{0}<T$. Sea $Y_{i t}^{I}$ el resultado que debería ser observado para la unidad $i$ en el periodo $t$ si la unidad $i$ es expuesta a la intervención en los periodos $T_{0}+1$ a $T$. Se asume que la intervención no tiene efecto sobre el resultado antes del periodo de implementación, esto es, para $t \in\left\{1, \ldots, T_{0}\right\}$ e $i \in\{1, \ldots, N\}$, tenemos que $Y_{i t}^{I}=Y_{i t}^{N 10}$. Adicionalmente, un supuesto implícito en esta metodología es la no interferencia entre unidades, esto es, asumimos que los resultados de las unidades no tratadas no son afectados por la intervención implementada en la unidad tratada (Rosenbaum, 2007).

Sea $\alpha=Y_{i t}^{I}-Y_{i t}^{N}$ el efecto de la intervención para la unidad $i$ en el periodo $t$, y sea $D_{i t}$ un indicador que toma el valor uno si la unidad $i$ es expuesta a la intervención en el periodo $t, \mathrm{y}$ cero en otro caso. El resultado observado para la unidad $i$ en el periodo $t$ es

$$
Y_{i t}=Y_{i t}^{N}+\alpha_{i t} D_{i t}
$$

Dado que la primer provincia es expuesta a la intervención y sólo después del periodo $T_{0}$

\footnotetext{
${ }^{9}$ Otras aplicaciones del MCS realizadas por estos autores incluyen la evaluación de un programa de control de tabaco a gran escala en California (Abadie et al., 2010) y el estudio del impacto económico de la reunificación alemana sobre Alemania Occidental (Abadie et al., 2014). Esta metodología ha sido utilizada también para analizar los efectos de las leyes de salario mínimo sobre el desempleo y salarios (Dube and Zipperer, 2013; Sabia et al., 2012), el rol desempeñado por las leyes migratorias sobre la composición poblacional (Bohn et al., 2014), entre otras políticas y programas implementados a nivel de un solo estado o jurisdicción.

${ }^{10}$ En la práctica, las intervenciones podrían tener un impacto antes de su implementación, por ejemplo, vía efecto anticipación. En estos casos, $T_{0}$ podría ser redefinido para ser el primer periodo en el cual el resultado podría responder a la intervención.
} 
$\left(\right.$ con $\left.1 \leq T_{0}<T\right)$, tenemos que

$$
D_{i t}= \begin{cases}1 & \text { si } i=1 \mathrm{y} t>T_{0} \\ 0 & \text { en otro caso. }\end{cases}
$$

Nuestro objetivo es estimar $\left(\alpha_{1 T_{0}+1}, \ldots, \alpha_{1 T}\right)$. Para $t>T_{0}$,

$$
\alpha_{1 t}=Y_{1 t}^{I}-Y_{1 t}^{N}=Y_{1 t}-Y_{1 t}^{N}
$$

Dado que $Y_{1 t}^{I}$ es observado, para estimar $\alpha_{1 t}$ resulta necesario estimar $Y_{1 t}^{N}$. Supongamos que $Y_{i t}^{N}$ es representado por el siguiente modelo

$$
Y_{i t}^{N}=\delta_{t}+\boldsymbol{\theta}_{t} \boldsymbol{Z}_{i}+\boldsymbol{\lambda}_{t} \boldsymbol{\mu}_{t}+\varepsilon_{i t}
$$

donde $\delta_{t}$ es un factor desconocido que es común y constante entre provincias, $\boldsymbol{Z}_{i}$ es un vector de variables de control, $\boldsymbol{\theta}_{t}$ es un vector de parámetros desconocidos, $\boldsymbol{\lambda}_{t}$ es un vector de factores no observables y comunes entre provincias, $\boldsymbol{\mu}_{t}$ es un vector de factores comunes desconocidos, y los términos de error $\varepsilon_{i t}$ son shocks transitorios no observables a nivel de las provincias con media cero.

Consideramos un vector $J x 1$ de ponderadores $\boldsymbol{W}=\left(w_{2}, \ldots, w_{J+1}\right)^{\prime}$ tal que $w_{j} \geq 0$ para $j=2, \ldots, J+1$, y $w_{2}+\ldots+w_{J+1}=1$. Cada valor particular del vector $\boldsymbol{W}$ representa un potencial control sintético, esto es, un promedio ponderado específico de provincias de control. $\mathrm{El}$ valor de la variable de resultado para cada control sintético indiciado por $\boldsymbol{W}$ es

$$
\sum_{j=2}^{J+1} w_{j} Y_{j t}=\delta_{t}+\boldsymbol{\theta}_{t} \sum_{j=2}^{J+1} w_{j} \boldsymbol{Z}_{j}+\boldsymbol{\lambda}_{t} \sum_{j=2}^{J+1} w_{j} \boldsymbol{\mu}_{j}+\sum_{j=2}^{J+1} w_{j} \varepsilon_{j t} .
$$

Suponemos que existe un $\left(w_{2}^{*}, \ldots, w_{J+1}^{*}\right)$ tal que

$$
\begin{aligned}
& \sum_{j=2}^{J+1} w_{j}^{*} Y_{j 1}=Y_{11}, \quad \sum_{j=2}^{J+1} w_{j}^{*} Y_{j 2}=Y_{12}, \quad \ldots, \\
& \sum_{j=2}^{J+1} w_{j}^{*} Y_{j 0}=Y_{1 T_{0}}, \quad y \quad \sum_{j=2}^{J+1} w_{j}^{*} \boldsymbol{Z}_{j}=\boldsymbol{Z}_{1} .
\end{aligned}
$$

Abadie et al. (2010) muestran que si $\sum_{t=1}^{T_{0}} \boldsymbol{\lambda}_{t}^{\prime} \boldsymbol{\lambda}_{t}$ es no singular, luego,

$$
Y_{1 t}^{N}-\sum_{j=2}^{J+1} w_{j}^{*} Y_{j t}=\sum_{j=2}^{J+1} w_{j}^{*} \sum_{s=1}^{T_{0}} \boldsymbol{\lambda}_{t}\left(\sum_{n=1}^{T_{0}} \boldsymbol{\lambda}_{n}^{\prime} \boldsymbol{\lambda}_{n}\right)^{-1}
$$




$$
\lambda_{s}^{\prime}\left(\varepsilon_{j s}-\varepsilon_{1 s}\right)-\sum_{j=2}^{J+1} w_{j}^{*}\left(\varepsilon_{j t}-\varepsilon_{1 t}\right) .
$$

Bajo condiciones estándar, el lado derecho de la ecuación (3) será cercano a cero si el número de periodos pre-intervención es grande en relación a la escala de los shocks transitorios. Esto sugiere utilizar $\widehat{\alpha}_{1 t}=Y_{1 t}-\sum_{j=2}^{J+1} w_{j}^{*} Y_{j t}$ para $t \in\left\{T_{0}+1, \ldots, T\right\}$ como un estimador de $\alpha_{1 t}$.

\subsection{Implementación}

Sea $\boldsymbol{W}=\left(w_{2}, \ldots, w_{J+1}\right)^{\prime}$ un vector $(J \times 1)$ de ponderadores positivos que suman la unidad. Esto es, $\boldsymbol{W}=\left(w_{2}, \ldots, w_{J+1}^{\prime}\right.$ con $w_{j} \geq 0$ para $j=2, \ldots, J+1$ y $w_{2}+\ldots+w_{J+1}=1$. Cada valor de $\boldsymbol{W}$ representa un promedio ponderado de provincias de control disponibles y, por tanto, un control sintético.

La variable de resultado de interés es observada durante $T$ periodos, $t=1, \ldots, T$, para la provincia afectada por la intervención, $Y_{1 t}$, y aquellas provincias no tratadas, $Y_{j t}, j=2, \ldots, J+1$. Sea el vector $\boldsymbol{K}=\left(k_{1}, \ldots, k_{T_{0}}\right)^{\prime}$ de $\left(T_{0} \times 1\right)$ el que define una combinación lineal de resultados pre-intervención: $\bar{Y}_{i}^{\boldsymbol{K}}=\sum_{s=1}^{T_{0}} k_{s} Y_{i s}$. Si $k_{1}=\ldots=k_{T_{0}-1}=0$ y $k_{T_{0}}=1$, luego $\bar{Y}_{i}^{\boldsymbol{K}}=Y_{i, T_{0}}$, el valor de la variable de resultado en el periodo inmediatamente anterior a la intervención. Por ejemplo, si $k_{1}=\ldots=k_{T_{0}}=1 / T_{0}$, luego, $\bar{Y}_{i}^{\boldsymbol{K}}=Y_{0}^{-1} \sum_{s=1}^{T_{0}} Y_{i s}$, el promedio simple de la variable de resultado en los periodos pre-intervención. Consideramos $M$ de tales combinaciones lineales definidas por los vectores $\boldsymbol{K}_{1}, \ldots, \boldsymbol{K}_{M}$. Sea $\boldsymbol{X}_{1}=\left(\boldsymbol{Z}_{1}^{\prime}, \bar{Y}_{1}^{\boldsymbol{K} 1}, \ldots, Y_{1}^{\boldsymbol{K}_{M}}\right)$ un vector $(k \mathrm{x} 1)$ de características pre-intervención para la provincia expuesta, con $k=r+M$. Similarmente, $\boldsymbol{X}_{0}$ es una matriz $(k \times J)$ que contiene las mismas variables para las provincias no afectadas. Esto es, la $j$-ésima columna de $\boldsymbol{X}_{0}$ es $\left(\boldsymbol{Z}_{j}^{\prime}, \bar{Y}_{j}^{\boldsymbol{K}_{1}}, \ldots, \bar{Y}_{j}^{\boldsymbol{K}_{M}}\right)$. El vector $\boldsymbol{W}^{*}$ es elegido para minimizar una distancia $\left\|\boldsymbol{X}_{1}-\boldsymbol{X}_{0} \boldsymbol{W}\right\|$, entre $\boldsymbol{X}_{1}$ y $\boldsymbol{X}_{0} \boldsymbol{W}$, sujeto a $w_{2} \geq 0, \ldots, w_{J+1} \geq 0$ y $w_{2}+\ldots+w_{J+1}=1$.

Para medir la discrepancia entre $\boldsymbol{X}_{1}$ y $\boldsymbol{X}_{0} \boldsymbol{W}$, emplearemos

$$
\left\|\boldsymbol{X}_{1}-\boldsymbol{X}_{0} \boldsymbol{W}\right\|_{\boldsymbol{V}}=\sqrt{\left(\boldsymbol{X}_{1}-\boldsymbol{X}_{0} \boldsymbol{W}\right)^{\prime} \boldsymbol{V}\left(\boldsymbol{X}_{1}-\boldsymbol{X}_{0} \boldsymbol{W}\right)}
$$

donde $\boldsymbol{V}$ es una matriz $(k \mathrm{x} k)$ semidefinida positiva y simétrica, aunque otras elecciones son posibles.

Aunque el procedimiento de inferencia es válido para cualquier elección de $\boldsymbol{V}$, esta última influye en el error cuadrático medio del estimador. Una elección óptima de $\boldsymbol{V}$ asigna ponderadores a la combinación lineal de las variables en $\boldsymbol{X}_{0}$ y $\boldsymbol{X}_{1}$ de manera tal que minimice el error cuadrático medio del estimador de control sintético. Esta elección puede estar basada en valoraciones subjetivas del poder predictivo de las variables en $\boldsymbol{X}_{1}$ y $\boldsymbol{X}_{0}$, aunque esta elección 
también puede ser conducida por un procedimiento basado en los datos. Siguiendo a Abadie and Gardeazabal (2003) y Abadie et al. (2010), se escogerá a la matriz $\boldsymbol{V}$ de manera tal que la provincia de control sintético resultante aproxime de la mejor manera la trayectoria de la variable de resultado de la provincia tratada en los periodos pre-intervención.

\subsection{Alcances y Limitaciones}

En relación a los alcances y limitaciones del MCS, pueden mencionarse los siguientes (Abadie et al., 2010; Craig, 2015): i) Debido a que el MCS se basa en la comparación entre un aglomerado real y otro de control sintético, los métodos estándar de inferencia estadística dejan de ser apropiados para el análisis. Una aproximación alternativa propuesta por Abadie et al. (2010) consiste en utilizar placebos, o tests de falsación, en los que el aglomerado de intervención es intercambiado por cada uno de los aglomerados que forman parte de panel de donantes ("across units placebo"). De esta manera, se genera un control sintético para cada aglomerado, y las tendencias post intervención son comparadas para cada par de controles reales y sintéticos, creando así un conjunto de efectos placebo. La diferencia en las tendencias post intervención del aglomerado tratado y su correspondiente control sintético puede ser comparada con las diferencias del resto de los pares generados a modo de placebo, y un valor p puede ser calculado a partir de la proporción de efectos placebo que son menores al efecto estimado para la intervención. ii) La intervención debería ser de relevancia a nivel del aglomerado en el cual es implementada. Si sólo afecta a una pequeña proporción de la población, la diferencia entre el aglomerado de intervención y su control sintético podría subestimar el verdadero efecto del programa. iii) Como fuera mencionado anteriormente, el efecto de la intervención sólo puede estimado de manera adecuada si no hay otros eventos adicionales que afecten solamente al aglomerado de intervención (en nuestro caso, programas especiales de vivienda, etc.). iv) La intervención debería afectar la variable de resultado sólo en el aglomerado de intervención, esto es, si "contamina" algunos o todos los aglomerados de control el verdadero efecto de la intervención podría ser subestimado. v) Resulta necesario un buen ajuste entre las tendencias pre-tratamiento del aglomerado de intervención y su control sintético con la intención de minimizar la posibilidad de sesgo en la comparación post-intervención.

\section{Datos}

La variable de resultado del estudio estará representada por el porcentaje de individuos que habitan viviendas familiares de calidad inadecuada a nivel de los aglomerados relevados por la EPH. Este indicador dicotómico de vivienda de calidad inadecuada será construido a partir 
de considerar cuatro aspectos clave de la vivienda familiar presentes en la EPH: a) Falta de instalación de agua corriente, b) Ausencia de baño de uso exclusivo, c) Situación de hacinamiento (cuatro o más personas por cuarto), y d) Vivienda construida con materiales de mala calidad. Estas medidas permitirán identificar, bajo el enfoque de la unión, si un individuo habita una vivienda de calidad inadecuada o no, y colapsando este indicador a nivel de aglomerado se obtiene el porcentaje de individuos que habitan viviendas familiares de calidad inadecuada para tal unidad de análisis ${ }^{11}$. Así, la base de datos principal del estudio consistirá en un panel de datos, donde las unidades de observación serán los aglomerados relevados por la EPH durante el periodo 1997-2006, siendo el aglomerado San Luis - El Chorrillo el de intervención, y el año 2003 el de inicio del programa.

Adicionalmente, se propone un conjunto de variables que buscan captar diferentes aspectos que podrían influir en la calidad de las viviendas a nivel provincial, entre las que se encuentran: a) Producto Bruto Geográfico (PBG) constante per cápita, b) Porcentaje del Gasto público provincial en programas de vivienda en relación al Gasto público social total, c) Porcentaje de la población mayor de 18 años con educación superior (al menos secundaria), d) Porcentaje de la población que es propietaria de su vivienda, d) Tasa de empleo, y e) Coeficiente de Gini. Mientras la información del PBG es obtenida a partir de estimaciones de CEPAL y el Gasto Público provincial y sus respectivas aperturas proviene del Ministerio de Economía, el resto de las variables serán construidas a partir de la información provista por la EPH.

Es importante mencionar que la EPH como fuente de información presenta la limitación de que sólo provee datos para ciudades que poseen más de 100.000 habitantes. En el caso de la provincia de San Luis, la EPH releva información sobre el aglomerado San Luis - El Chorrillo, por lo que los resultados a obtener serán representativos del área urbana más poblada de la provincia, aplicando similares consideraciones para el resto de las jurisdicciones del país.

Por otra parte, y ante la posibilidad de explotar el Plebiscito sobre la continuidad del Plan de Inclusión Social a modo de experimento natural, se cuenta con información proveniente de registros históricos de la Cámara Argentina del Cemento, lo que posibilitará el armado de la proxy de consumo de cemento per cápita para cada una de las provincias argentinas para cada uno de los meses de 2004.

\footnotetext{
${ }^{11}$ Para el cálculo de la variable de resultado a nivel aglomerado se acumularán las diferentes ondas de relevamiento de la EPH de un mismo año, con la intención de contar con un mayor número de observaciones.
} 


\section{Resultados}

A continuación, la Figura 2 muestra las tendencias en el porcentaje de individuos que habitan viviendas de calidad inadecuada en el aglomerado San Luis y en el resto del país. Como la figura sugiere, el resto del país no constituye un buen grupo de control para estudiar el efecto del Plan de Inclusión Social en San Luis. Incluso antes de la implementación del programa el porcentaje de individuos que habitan viviendas familiares de calidad inadecuada difiere notablemente entre San Luis y el resto del país, siendo esta diferencia de aproximadamente 5 puntos porcentuales promedio por año pre-tratamiento. A partir de la implementación del programa, en el año 2003, el porcentaje de individuos que habitan viviendas de calidad inadecuada en el resto del país parece estabilizarse en un valor de aproximadamente $9.5 \%$, mientras que en San Luis tal indicador refuerza su caída, alcanzando valores cercanos al $5 \%$ a dos años del inicio del programa.

\section{[FIGURA 2]}

\subsection{Efecto del programa}

Para evaluar el impacto del programa de transferencias de ingresos Plan de Inclusión Social sobre la variable de resultado propuesta resulta clave responder cómo habría evolucionado tal variable en San Luis ante la ausencia del programa. En este sentido, el MCS nos permite construir dicho contrafactual.

Como fuera mencionado en la sección metodológica, se construirá un aglomerado San Luis sintético como una combinación convexa del resto de los aglomerados que repliquen adecuadamente a San Luis en términos de los valores pre-intervención de los predictores de la variable de interés. Los resultados de este procedimiento son presentados en la Tabla 1, que compara las características pre-tratamiento del aglomerado San Luis real y su contraparte sintética. Se observa que el aglomerado San Luis sintético reproduce de una manera adecuada tanto el porcentaje de individuos que habitan viviendas inadecuadas como los predictores propuestos antes de la implementación del programa, con excepción del predictor Gasto Público en Vivienda/Gasto Público Social total, que en la unidad tratada es superior durante el periodo pre-tratamiento ${ }^{12}$. De cualquier manera, como se verá más adelante, esta variable no parece tener peso significativo en la construcción de San Luis sintético.

\footnotetext{
${ }^{12}$ Como fuera mencionado anteriormente, en términos presupuestarios los principales "aportantes" para el funcionamiento del Plan de Inclusión Social fueron las partidas tradicionalmente destinadas a obra pública, entre las que se destacan las asignadas a construcción de viviendas. Ello llevó a que el gasto público provincial en programas de vivienda, expresado en términos del gasto público social, pasara de representar más del $17 \%$ promedio en 1996-2002 a sólo un 5.5\% promedio en el periodo 2004-2006, esto es, antes y después de la implementación del programa.
} 


\section{[TABLA 1]}

La Tabla 2 muestra el ponderador que corresponde a cada aglomerado del panel de donantes en el armado del aglomerado San Luis sintético. Estos ponderadores indican que la tendencia en el porcentaje de individuos que habitan viviendas de calidad inadecuada antes de la implementación del programa es reproducida de la mejor manera por una combinación convexa de los aglomerados Río Cuarto, Gran Rosario, Gran Resistencia, Ciudad de Buenos Aires y Concordia. El resto de los aglomerados en el panel de donantes asumen un ponderador igual a cero (como fuera mencionado anteriormente, los aglomerados Neuquén-Plottier y Santa Rosa-Toay fueron excluidos del análisis por poseer una incidencia de programas sociales provinciales no despreciable).

[TABLA 2]

A continuación, la Figura 3 muestra el porcentaje de individuos que habitan viviendas familiares de calidad inadecuada tanto para el aglomerado San Luis real como para su contraparte sintética durante el periodo 1996-2006. Se observa que, en contraste con la tendencia del indicador de resultado para el caso del resto del país (Figura 1), el porcentaje de individuos en viviendas de calidad inadecuada en San Luis sintético ajusta de muy buena manera la trayectoria del indicador en San Luis real durante todo el periodo pre-intervención. Esto, combinado con el alto grado de balance en los predictores propuestos (Tabla 1), sugiere que el aglomerado sintético constituye una muy buena representación de San Luis en ausencia del Plan de Inclusión Social.

\section{[FIGURA 3]}

El efecto del programa sobre la variable de interés es representado por la diferencia en las tendencias entre San Luis real y su contraparte sintética después de la implementación de la intervención. Se observa que, luego de dos años de haberse implementado el Plan de Inclusión Social, las tendencias en la variable de resultado entre ambas unidades comienzan a diverger. Si bien el porcentaje de individuos habitando viviendas de calidad deficiente continúa su tendencia decreciente tanto en San Luis real como en su contraparte sintética, es en la primer unidad donde esta tendencia a la baja se profundiza. La diferencia entre ambas líneas sugiere un efecto del Plan de Inclusión Social en el porcentaje de individuos que habitan viviendas de calidad inadecuada.

En esta dirección, la Figura 4 presenta las estimaciones de este efecto año a año, esto es, las brechas anuales en el porcentaje de individuos que habitan viviendas de calidad inadecuada en el aglomerado San Luis y su contraparte sintética. La figura sugiere que el efecto del programa 
sobre la variable de resultado se incrementa en el tiempo, al punto que en el año 2006 el porcentaje de individuos habitando viviendas de calidad inadecuada en San Luis se redujo en 1.85 puntos porcentuales, lo que representa una reducción relativa del $25.21 \%$ cuando se lo compara con la unidad de control.

[FIGURA 4]

\section{2. $\quad$ Test placebo}

Como fuera mencionado en el apartado metodológico, una de las limitaciones que posee el MCS es que los métodos estándar de inferencia estadística dejan de ser apropiados para evaluar la significancia del efecto estimado del programa. En línea con el trabajo de Abadie and Gardeazabal (2003), Abadie et al. (2010) y Bertrand et al. (2004), se realizará una serie de experimentos placebo consistentes en asignar artificialmente la intervención a cada uno de los aglomerados del panel de donantes y aplicar el MCS a cada uno de ellos. Si a partir de este procedimiento se obtienen efectos cuya magnitud sea igual o superior al identificado para el aglomerado San Luis, estaremos en condiciones de decir que no disponemos de evidencia significativa de que el Plan de Inclusión Social haya posibilitado una reducción en el porcentaje de individuos que habitan viviendas de calidad inadecuada. Por el contrario, si los efectos estimados son inferiores al hallado en San Luis diremos que el efecto estimado del programa es estadísticamente significativo.d

La Figura 5 presenta los resultados del experimento descripto anteriormente. Cada línea de color gris representa el efecto simulado del programa para cada uno de los aglomerados del panel de donantes durante el periodo bajo análisis, mientras que la línea negra superpuesta muestra el efecto estimado del programa en el aglomerado San Luis. La figura muestra el buen ajuste del control sintético para el caso de San Luis durante el periodo pre-tratamiento, la correcta dirección del efecto del programa y la magnitud creciente del mismo en relación a los placebos.

\section{[FIGURA 5]}

El error cuadrático medio de predicción en San Luis durante el periodo pre-intervención es de 0.12 , mientras que tal valor para el promedio de los aglomerados del panel de donantes fue de 0.48 , sugiriendo que el MCS provee un buen ajuste tanto para la unidad tratada como para los placebos. Sin embargo, la figura sugiere la existencia de un grupo de aglomerados placebo para los cuales el comportamiento de la variable de resultado no puede ser replicado de manera adecuada por la combinación convexa de otros aglomerados durante el periodo pre- 
intervención, por lo que estos placebos no proporcionarían información de utilidad para medir la significatividad del efecto del programa en el aglomerado San Luis.

A partir de ello, la Figura 6 conserva solamente a los aglomerados placebo que poseen un error cuadrático medio de predicción durante el periodo pre-intervención que sea inferior a 10 veces al que corresponde al aglomerado San Luis, descartando así a 16 aglomerados placebo. Entre los 10 aglomerados presentes en la figura, la línea que corresponde al aglomerado San Luis se configura como la más inusual en términos de dirección y magnitud del efecto durante el periodo post-intervención. Debido a que la figura incluye 10 aglomerados de control, la probabilidad de estimar un efecto de la magnitud obtenida para el aglomerado San Luis bajo una asignación aleatoria de la intervención es de $10 \%$, un nivel que resulta de uso convencional en los tests de significancia estadística.

\section{[FIGURA 6]}

\subsection{Desagregación del indicador de calidad de la vivienda familiar}

El indicador de calidad inadecuada de la vivienda familiar analizado previamente resulta útil como variable de resultado, pero al tratarse de un indicador que sintetiza diferentes atributos de la vivienda familiar (sub-indicadores de falta de conexión a agua corriente, baño de uso exclusivo, hacinamiento y materiales de mala calidad), su interpretación podría ser algo limitada. Esto es, la reducción en el porcentaje de individuos que habitan viviendas de calidad inadecuada podría haber operado a través de la mejora de uno o varios sub-indicadores de calidad de la vivienda.

Resulta claro que la mejora de cada uno de estos atributos difiere tanto en costos como en posibilidades de acceso para las familias. Por ejemplo, la mejora de los materiales con los que la vivienda fue construida podría ser menos costosa que la construcción de un cuarto adicional para reducir el hacinamiento. De la misma manera, la mejora en cualquiera de estos sub-indicadores debería ser más factible que lograr para el hogar que poseer baño de uso exclusivo dentro de la vivienda, ya que este último atributo presupone la inversión inicial por parte del gobierno proveyendo servicios de saneamiento.

Bajo este marco, se procede a desagregar el indicador propuesto de calidad inadecuada de la vivienda familiar a nivel de los sub-indicadores que lo componen (falta de agua corriente, falta de baño de uso exclusivo, hacinamiento y materiales de mala calidad), para aplicar el MCS en términos del porcentaje de individuos que habitan viviendas que carecen de cada uno de estos atributos. La Figura 7 presenta los resultados que surgen de realizar tal ejercicio.

[FIGURA 7] 
Se observa que el programa provincial de transferencias de ingresos posibilitó una mejoría en los sub-indicadores de menor costo y mayor posibilidad de acceso para el hogar, como es el caso de la conexión a agua corriente y mejora de los materiales de construcción, no así en la tenencia de baño de uso exclusivo dentro de la vivienda, posiblemente el sub-indicador de mayor costo y dificultad de acceso. Este resultado parece apoyar el mecanismo propuesto a través del cual operaría el programa, esto es, la asignación parcial -por parte de las familias- de las transferencias recibidas a la mejora de la calidad de la vivienda que habitan.

\section{Utilizando la Consulta Popular sobre la Continuidad del Plan de Inclusión Social como Experimento Natural}

De manera adicional al desarrollado anteriormente, se propone explotar -a modo de experimento natural- un suceso político ocurrido en la provincia de San Luis durante el año 2004, con la intención de proveer evidencia sobre el canal a través del cual el programa podría haber reducido el porcentaje de individuos que habitan viviendas familiares de calidad inadecuada.

A partir de la implementación del Plan de Inclusión Social en mayo de 2003 comenzó un proceso de descontento social generalizado en la provincia con el gobierno electo. Este descontento social tuvo diversas causas, entre las que se encuentran el desconocimiento por parte del gobierno provincial de las elecciones municipales de la ciudad de San Luis del 9 de noviembre de ese mismo año, en la que fue electo un dirigente opositor, los reclamos docentes por el pago de deudas salariales y mejoras edilicias en marzo de 2004, que resultaron en un paro del ciclo lectivo, el rechazo de la iglesia católica a la intervención realizada por el gobierno provincial en los institutos autoadministrados por esa congregación, entre otras. Estos factores llevaron a la realización de numerosas marchas y cacerolazos masivos en marzo de 2004 exigiendo la renuncia del gobernador, obteniendo por respuesta una serie de contramarchas oficialistas, generándose así una delicada situación política, social e institucional en la provincia.

Ante este contexto adverso, el gobierno provincial decidió anunciar el 22 de abril de 2004, mediante la Ley provincial 5593, la convocatoria a una consulta popular (plebiscito) para decidir por la continuidad o interrupción del Plan de Inclusión Social. Esta consulta popular fue convocada para el 22 de agosto de ese mismo año, y el voto tuvo carácter obligatorio, con efecto vinculante, y debía cumplir con todos los requisitos establecidos por la Ley electoral vigente. La iniciativa de la consulta popular fue rechazada de lleno por el arco opositor de la provincia, en tanto la consideraron innecesaria, habida cuenta de que el programa social de ayuda a los desocupados no se encontraba en el centro de los cuestionamientos al gobierno. Esto llevó a que 
en los meses previos a la consulta popular no hubiera una campaña a favor de la interrupción del programa, de la misma manera que no hubo fiscales por tal opción el día de la elección. El resultado de la misma fue una adhesión de la ciudadanía de San Luis a la continuidad del programa social provincial.

Si efectivamente el Plan de Inclusión Social llevó a la población beneficiaria a destinar parte de sus ingresos a la mejora de la vivienda familiar, entonces sucesos como la convocatoria por ley a la consulta popular, así como la falta de una campaña a favor de la interrupción del programa, y la posterior convalidación del mismo en la urnas, deberían haber tenido efecto en las decisiones de compra de materiales para la construcción en la provincia. Esto es, sería de esperar una reducción en la compra de materiales en los meses de abril y mayo (anuncio del plebiscito), una ligera recuperación de las mismas en junio y julio (dada la falta de campaña a favor de la interrupción del Plan antes de la elección), y un incremento significativo en las compras de materiales en los meses de septiembre y octubre (meses posteriores a la convalidación del programa social en las urnas).

Estas decisiones de compra de materiales para la construcción serán representadas por una variable proxy de consumo de cemento per cápita mensual a nivel provincia, construida a partir de registros históricos de la Cámara Argentina del Cemento, disponible para cada mes de 2004 y para cada provincia del país. La Figura 8 presenta la evolución temporal de este indicador de consumo de cemento per cápita para la provincia de San Luis a lo largo de 2004, la cual parecería responder al patrón de comportamiento propuesto.

\section{[FIGURA 8]}

Debido a que otros factores podrían estar explicando este resultado (posible estacionalidad en la demanda de cemento, heterogeneidad no observada a nivel de provincias, etc.), la hipótesis de comportamiento propuesta será testeada a partir de la estimación de un modelo econométrico que permite controlar por efectos fijos a nivel de mes y provincia, y que adopta la siguiente especificación:

$$
Y_{i t}=\alpha+\beta \text { SanLuis }+\gamma M e s_{t}+\varphi \text { SanLuis } * M e s_{t}+\varepsilon_{i t}
$$

en donde la variable dependiente $Y_{i t}$ representa la proxy de consumo de cemento per cápita en la provincia $i$ en el mes $t$, SanLuis es una variable dummy que adopta el valor 1 para dicha provincia y 0 para cualquier otra, Mes representa una variable dummy para cada uno de los meses $t$ del año, y pretende capturar efectos estacionales en el consumo de cemento, y SanLuis * Mes es un set de interacciones que buscan identificar comportamientos específicos 
del consumo per cápita de cemento en la provincia de San Luis en los diferentes meses $t$ del año, siendo $\varepsilon_{i t}$ el término error, que se asume cumple los supuestos clásicos. Los resultados de esta estimación son presentados en la Tabla 3.

\section{[TABLA 3]}

Tanto la estimación realizada mediante Efectos Fijos (columna 1) como a través de Efectos Aleatorios (columna 2) parecen validar la hipótesis de comportamiento propuesta, esto es, la caía del consumo de cemento per cápita en San Luis en el mes de mayo (anuncio del plebiscito), así como su recuperación en los meses posteriores a agosto (convalidación del programa en las urnas) son estadísticamente significativas aún cuando se controla por efectos estacionales (meses del año) y locacionales (efectos fijos a nivel provincia), apoyando el canal propuesto a través del cual el programa redujo el porcentaje de individuos que habitan viviendas de calidad inadecuada.

\section{Conclusiones}

El presente trabajo ha tenido por objetivo general contribuir a la incipiente literatura sobre los efectos indirectos de los programas sociales en términos del uso de las transferencias por parte de las familias. Para ello, se evaluó el efecto del Plan de Inclusión Social, implementado en la provincia de San Luis en 2003, sobre un indicador que posee implicancias en el bienestar de mediano y largo plazo de los hogares: la calidad de la vivienda familiar.

Los resultados obtenidos sugieren un efecto del programa en la reducción del porcentaje de individuos que habitan viviendas de calidad inadecuada de 1.85 puntos porcentuales a dos años de su implementación. Si bien este efecto podría parecer modesto, representa una reducción relativa de $25.21 \%$ cuando se lo compara con el control sintético, y es robusto a una serie de pruebas de falsación. El canal propuesto para este efecto, es decir, la decisión de los hogares de destinar al menos una parte de las transferencias a la mejora de la calidad de la vivienda familiar, parece ser apoyado por los resultados que surgen de explotar, a modo de experimento natural, el plebiscito al que fue sometido el programa en 2004.

Se espera que los resultados presentados en este trabajo contribuyan al debate informado sobre los efectos de bienestar que podrían tener estos programas más allá de las condicionalidades requeridas para su percepción, brindando apoyo adicional para su fortalecimiento como instrumento de protección social para la población vulnerable. 


\section{Referencias}

Abadie, A., Diamond, A., and Hainmueller, J. (2010). Synthetic Control Methods for Comparative Case Studies: Estimating the Effect of California's Tobacco Control Program. Journal of the American Statistical Association, 105(490):493-505.

Abadie, A., Diamond, A., and Hainmueller, J. (2014). Comparative Politics and the Synthetic Control Method. American Journal of Political Science, 59(2):495-510.

Abadie, A. and Gardeazabal, J. (2003). The Economic Costs of Conflict: A Case Study of the Basque Country. American Economic Review, 93(1):113-132.

Aiga, H., Arai, Y., Marui, E., and Umenai, T. (1999). Impact of Improvement of Water Supply on Reduction of Diarrheal Incidence in a Squatter Area of Manila. Environmental Health and Preventive Medicine, 4(3):111-116.

Alzúa, M. L., Cruces, G., and Ripani, L. (2013). Welfare Programs and Labor Supply in Developing Countries: Experimental Evidence from Latin America. Journal of Population Economics, 26(4):1255-1284.

Angelucci, M. and Attanasio, O. (2013). The Demand for Food of Poor Urban Mexican Households: Understanding Policy Impacts Using Structural Models. American Economic Journal: Economic Policy, 5(1):146-205.

Angelucci, M. and De Giorgi, G. (2009). Indirect Effects of an Aid Program: How Do Cash Transfers Affect Ineligibles' Consumption? The American Economic Review, 99(1):486-508.

Ashenfelter, O. (1983). Determining Participation in Income-tested Social Programs. Journal of the American Statistical Association, 78(383):517-525.

Attanasio, O. P., Bottazzi, R., Low, H. W., Nesheim, L., and Wakefield, M. (2012). Modelling the Demand for Housing over the Life Cycle. Review of Economic Dynamics, 15(1):1-18.

Banerjee, A. and Mullainathan, S. (2010). The Shape of Temptation: Implications for the Economic Lives of the Poor. National Bureau of Economic Research, 15973.

Banerjee, A. V., Hanna, R., Kreindler, G., and Olken, B. A. (2015). Debunking the Stereotype of the Lazy Welfare Recipient: Evidence from Cash Transfer Programs Worldwide. Harvard Kennedy School Working Paper, 308.

Barrientos, A. (2012). Social Transfers and Growth: What Do We Know? What Do We Need to Find Out? World Development, 40(1):11-20. 
Bertrand, M., Duflo, E., and Mullainathan, S. (2004). How Much Should We Trust Differencesin-Differences Estimates? Quarterly Journal of Economics, 119(1):249-275.

Bohn, S., Lofstrom, M., and Raphael, S. (2014). Did the 2007 Legal Arizona Workers Act Reduce the State's Unauthorized Immigrant Population? Review of Economics and Statistics, 96(2):258-269.

Bussetti, M. (2009). Inclusión Social ¿En qué? ¿Para qué? Un Estudio de Caso. XXVII Congreso de la Asociación Latinoamericana de Sociología, Buenos Aires, Argentina.

Cattaneo, M. D., Galiani, S., Gertler, P. J., Martinez, S., and Titiunik, R. (2009). Housing, Health, and Happiness. American Economic Journal: Economic Policy, 1(1):75-105.

Choudhary, R., Sharma, A., Agarwal, K. S., Kumar, A., Sreenivas, V., and Puliyel, J. M. (2002). Building for the Future: Influence of Housing on Intelligence Quotients of Children in an Urban Slum. Health Policy and Planning, 17(4):420-424.

Conconi, A. (2011). Pobreza Multidimensional en Argentina: Ampliando las Medidas Tradicionales de Pobreza por Ingresos y NBI. Departamento de Economía UNLP, Serie Documentos de Trabajo, 90 .

Cruces, G. and Gasparini, L. (2008). Programas Sociales en Argentina: Alternativas para la Ampliación de la Cobertura. Documentos de Trabajo CEDLAS, 77.

Cruces, G., Moreno, J. M., Ringold, D., and Rofman, R. (2008). Los Programas Sociales en Argentina hacia el Bicentenario. Visiones y Perspectivas. Banco Mundial.

Dasso, R. and Fernández, F. (2013). Temptation Goods and Conditional Cash Transfers in Perú. Unpublished manuscript.

DGPyPS (2006). Informe sobre Programas de Empleo Provinciales. Serie Gasto Público, Documento de Trabajo, 17.

Dorzán, M. and Arias, L. (2005). De una Simple Mirada... A una Mirada no tan Simple... El Plan de Inclusión Social, Trabajo por San Luis. Kayros. Revista de Temas Sociales, 15.

Dube, A. and Zipperer, B. (2013). Pooled Synthetic Control Estimates for Recurring Treatments: An Application to Minimum Wage Case Studies. University of Massachusetts, Amherst.

Evans, D. and Popova, A. (2016). Cash Transfers and Temptation Goods. Economic Development and Cultural Change (Forthcoming). 
Feigelman, D. (2008). Desde el Enfoque de la Economía Social, Comprender las Desventajas Sociales para Poder Paliarlas. San Luis, ¿Una Política Social Diferente? V Jornadas de Sociología de la Universidad Nacional de La Plata, Buenos Aires, Argentina.

Fiszbein, A., Schady, N. R., Ferreira, F. H., Grosh, M., Keleher, N., Olinto, P., and Skoufias, E. (2009). Conditional Cash Transfers: Reducing Present and Future Poverty. World Bank Publications.

Galasso, E. and Ravallion, M. (2004). Social Protection in a Crisis: Argentinaś Plan Jefes y Jefas. The World Bank Economic Review, 18(3):367-399.

Galiani, S., Gertler, P., Cooper, R., Martinez, S., Ross, A., and Undurraga, R. (2016). Shelter From the Storm: Upgrading Housing Infrastructure in Latin American Slums. Journal of Urban Economics, 96:166-194.

Galiani, S., Gertler, P., and Schargrodsky, E. (2005). Water for Life: The Impact of the Privatization of Water Services on Child Mortality. Journal of Political Economy, 113(1):83-120.

Gertler, P. J., Martinez, S. W., and Rubio-Codina, M. (2012). Investing Cash Transfers to Raise Long-term Living Standards. American Economic Journal: Applied Economics, 4(1):164-192.

Glassman, A., Duran, D., Fleisher, L., Singer, D., Sturke, R., Angeles, G., Charles, J., Emrey, B., Gleason, J., Mwebsa, W., et al. (2013). Impact of Conditional Cash Transfers on Maternal and Newborn Health. Journal of Health, Population, and Nutrition, 31(4 Suppl 2):S48-S66.

Golbert, L. (2004). Derecho a la Inclusión o Paz Social? Plan Jefas y Jefes de Hogar Desocupados. CEPAL, Serie Políticas Sociales, 84.

Golbert, L. (2007). Aprendizajes del Programa de Jefes y Jefas de Argentina. En Arriagada, I. (Coordinadora), Familias y Políticas Públicas en América Latina: Una Historia de Desencuentros. CEPAL, Naciones Unidas.

Handa, S. and Davis, B. (2006). The Experience of Conditional Cash Transfers in Latin America and the Caribbean. Development Policy Review, 24(5):513-536.

Jacob, B., Kapustin, M., and Ludwig, J. (2014). Human Capital Effects of Anti-poverty Programs: Evidence from a Randomized Housing Voucher Lottery. National Bureau of Economic Research, 20164.

Maluccio, J. A. (2010). The Impact of Conditional Cash Transfers on Consumption and Investment in Nicaragua. The Journal of Development Studies, 46(1):14-38. 
Mas-Colell, A., Whinston, M. D., and Green, J. R. (1995). Microeconomic Theory. Oxford University Press New York.

Olguín, J. R., Becerra, M. E., Vilchez, V. C., and Ruiz, K. A. (2012). Mercado Laboral y Desarrollo Territorial. San Luis y Cuyo. Revista de Estudios Regionales y Mercado de Trabajo, $8: 31-56$.

Olguín, J. R., Busseti, M., and Páez, M. (2005). Desocupación, Pobreza y Planes Sociales en San Luis. Revista de Estudios Regionales y Mercado de Trabajo, 1:31-46.

Olguín, J. R. and Páez, M. (2005). Incidencia de los Planes Sociales en los Indicadores del Mercado Laboral San Luis 2003-2004, Un Caso Paradigmático. VII Congreso Nacional de Estudios del Trabajo, Buenos Aires, Argentina.

ONU-Hábitat (2010). El Derecho a una Vivienda Adecuada. Folletos Informativos sobre los Derechos Humanos, 21.

Páez, M., Bussetti, M., Saibene, M., and Fernández, M. C. (2008). Distribución Presupuestaria y Política Social en la Provincia de San Luis. II Jornadas Nacionales de Investigadores de las Economías Regionales, Tandil, Buenos Aires, Argentina.

Rawlings, L. B. and Rubio, G. M. (2005). Evaluating the Impact of Conditional Cash Transfer Programs. The World Bank Research Observer, 20(1):29-55.

Rosenbaum, P. R. (2007). Interference Between Units in Randomized Experiments. Journal of the American Statistical Association, 102(477):191-200.

Sabia, J., Burkhauser, R. V., and Hansen, B. (2012). Are the Effects of Minimum Wage Increases Always Small? New Evidence from a Case Study of New York State. Industrial and Labor Relations Review, 65(2):350-376.

Stecklov, G., Winters, P., Todd, J., and Regalia, F. (2007). Unintended Effects of Poverty Programmes on Childbearing in Less Developed Countries: Experimental Evidence from Latin America. Population Studies, 61(2):125-140.

Suárez Godoy, E. H. (2004). San Luis, Una Política Social Diferente. Plan de Inclusión Social. San Luis: Talleres Gráficos Payné.

Vilchez, V. (2013). Análisis de las Políticas Sociales desde la Perspectiva de los Derechos. El Caso del Plan de Inclusión Social "Trabajo por San Luis". Revista Electrónica de Psicología Política, 30:57-73. 
Vilchez, V. and Feigelman, D. (2009). Beneficiarios del Plan de Inclusión Social, ¿Ciudadanos Soberanos o Ciudadanos Siervos? V Jornadas de Jóvenes Investigadores. Instituto de Investigaciones Gino Germani, Buenos Aires, Argentina.

Wolff, C. G., Schroeder, D. G., and Young, M. W. (2001). Effect of Improved Housing on Illness in Children Under 5 Years Old in Northern Malawi: Cross Sectional Study. British Medical Journal, 322(7296):1209-1212. 


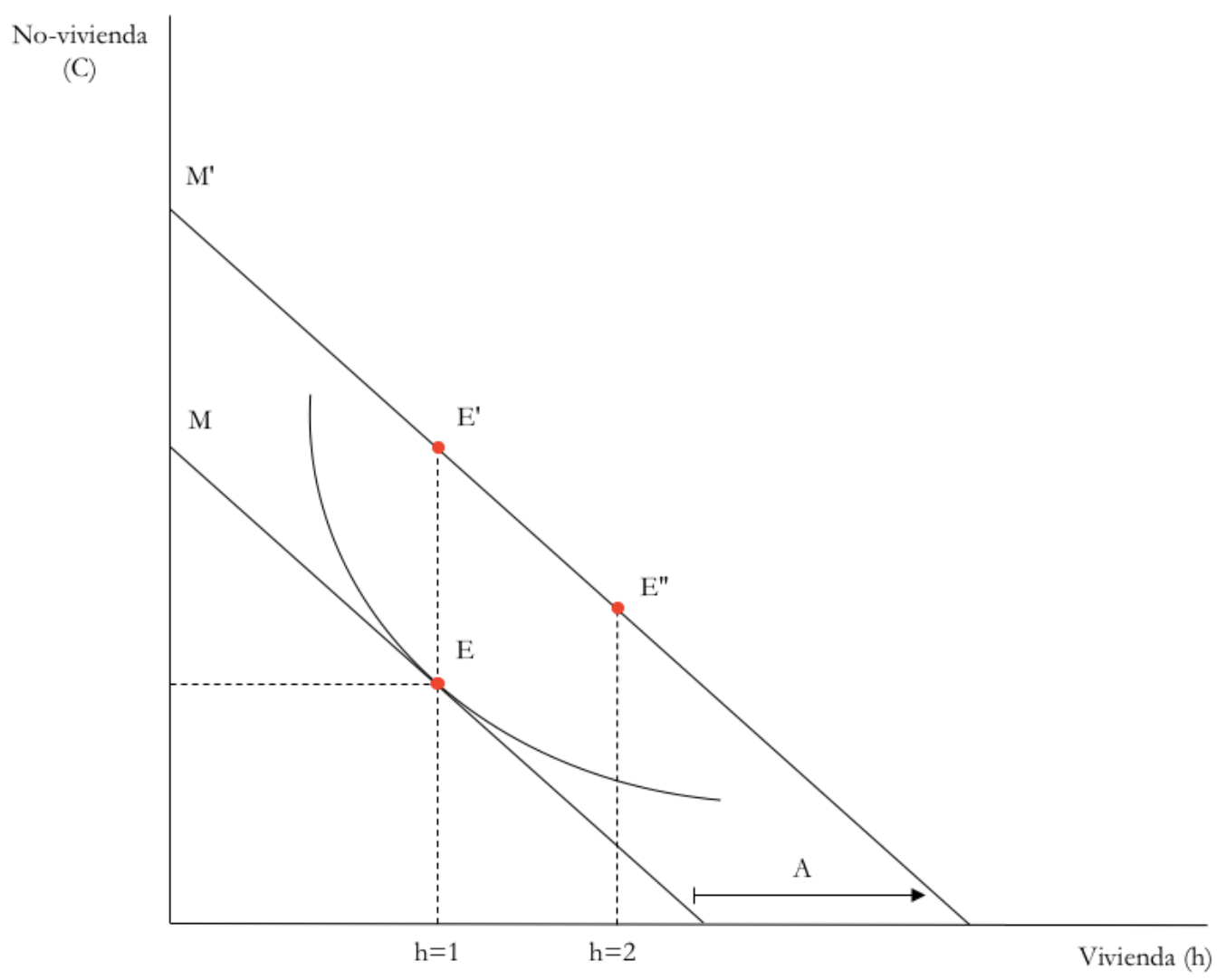

Figura 1: Restricción presupuestaria y espacio de consumo del hogar en situación de pobreza sin y con el programa de transferencia de ingresos.

Tabla 1: Características pre-tratamiento. Promedios 1996-2002.

\begin{tabular}{lcc}
\hline & \multicolumn{2}{c}{ San Luis } \\
\cline { 2 - 3 } & Real & Sintético \\
\hline Ln PBG per cápita & 10.24 & 10.14 \\
Gasto Público vivienda/GPS total \% & 17.88 & 3.38 \\
Pob. con educucación superior \% & 40.89 & 42.91 \\
Propiedad de la vivienda \% & 78.99 & 76.59 \\
Tasa de empleo \% & 33.41 & 34.78 \\
Coeficiente de Gini & 0.47 & 0.45 \\
Ind. en viviendas inadecuadas 1996\% & 16.08 & 16.08 \\
Ind. en viviendas inadecuadas 1999\% & 11.40 & 11.40 \\
Ind. en viviendas inadecuadas 2002\% & 7.69 & 7.71 \\
\hline
\end{tabular}




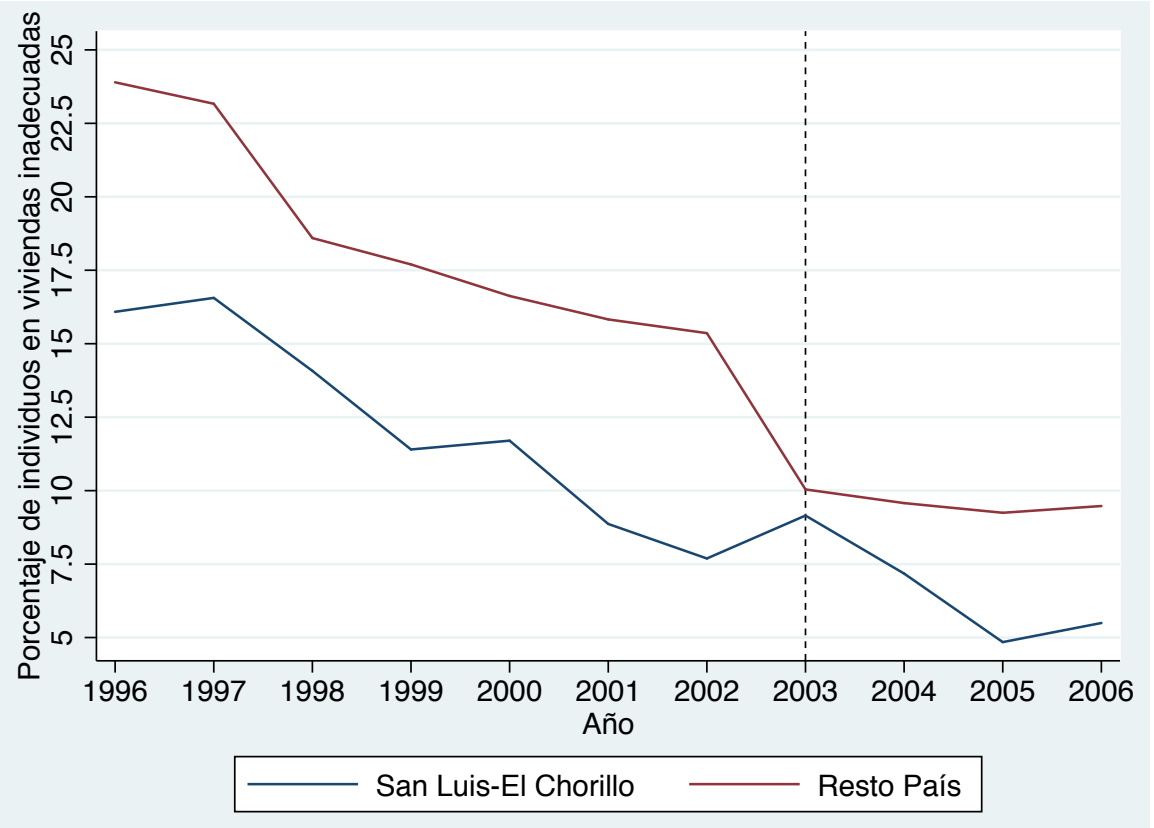

Figura 2: Tendencias en el porcentaje de individuos que habitan viviendas familiares inadecuadas: San Luis y Resto del País.

Tabla 2: Ponderadores de los aglomerados.

\begin{tabular}{lclc}
\hline Aglomerado & Ponderador & Aglomerado & Ponderador \\
\hline Gran La Plata & 0.00 & Santiago del Estero-La Banda & 0.00 \\
Bahía Blanca-Cerri & 0.00 & Jujuy-Palpalá & 0.00 \\
Gran Rosario & 0.14 & Río Gallegos & 0.00 \\
Gran Santa Fe & 0.00 & Gran Catamarca & 0.00 \\
Gran Paraná & 0.00 & Salta & 0.00 \\
Posadas & 0.00 & La Rioja & 0.00 \\
Gran Resistencia & 0.06 & Gran San Juan & 0.00 \\
Comodoro Rivadavia-Rada Tilly & 0.00 & Gran Tucumán-Tafí Viejo & 0.00 \\
Gran Mendoza & 0.00 & Santa Rosa-Toay & - \\
Corrientes & 0.00 & Ushuaia-Río Grande & 0.00 \\
Gran Córdoba & 0.00 & Ciudad de Buenos Aires & 0.20 \\
Concordia & 0.13 & Partidos del Conurbano & 0.00 \\
Formosa & 0.00 & Mar del Plata-Batán & 0.00 \\
Neuquén-Plottier & - & Río Cuarto & 0.47 \\
\hline
\end{tabular}




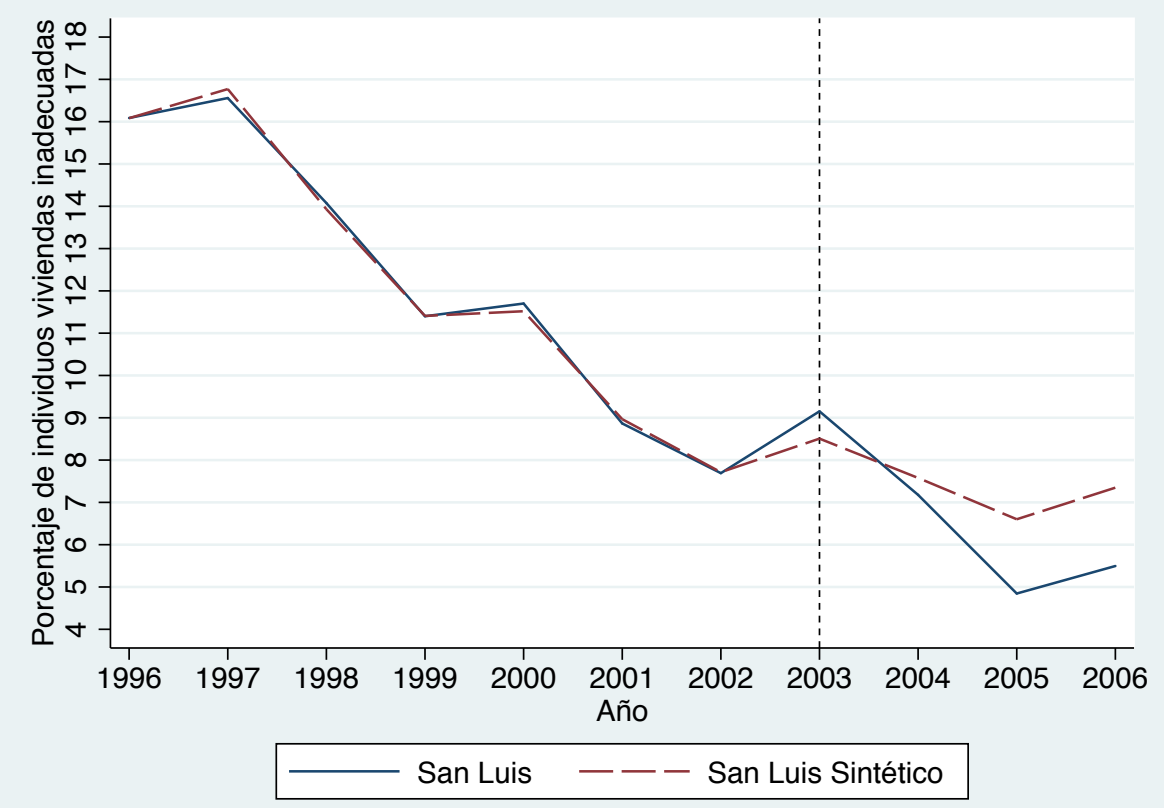

Figura 3: Tendencias en el porcentaje de individuos que habitan viviendas inadecuadas: San Luis Real y San Luis Sintético.

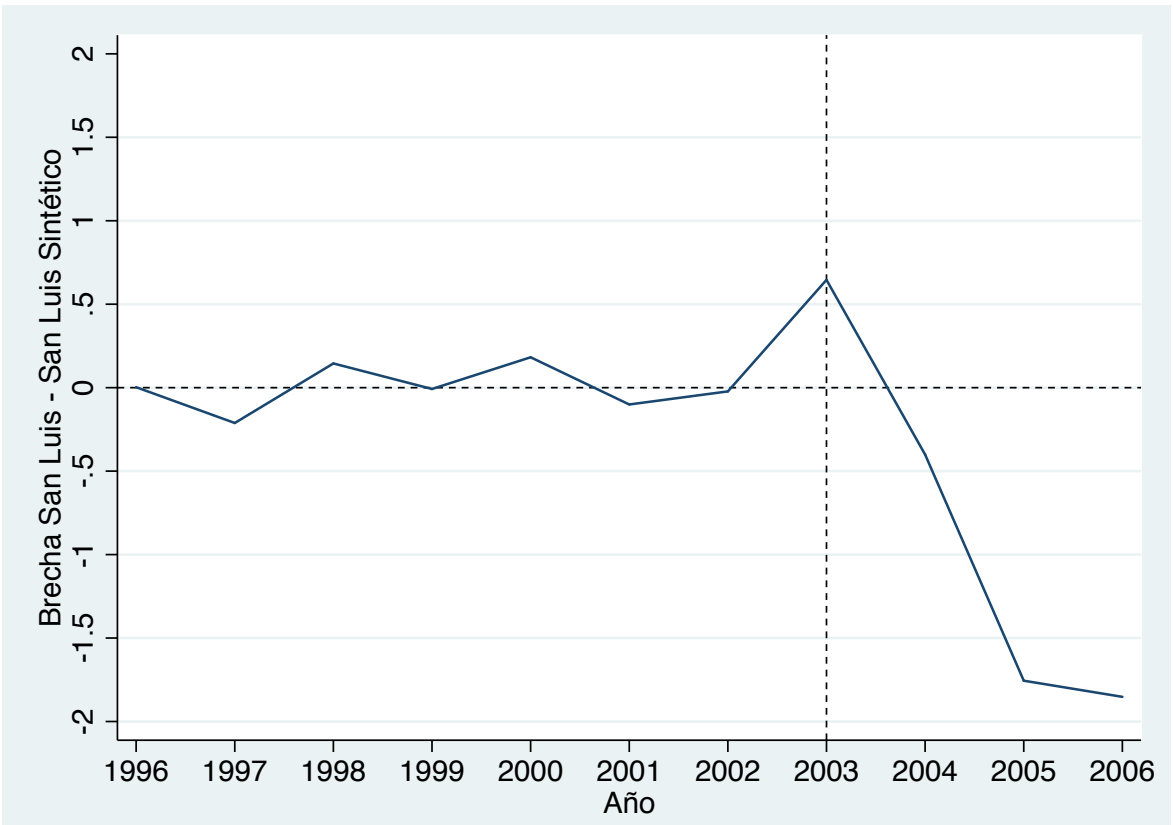

Figura 4: Brecha en el porcentaje de individuos que habitan viviendas inadecuadas a partir de la implementación del programa. 


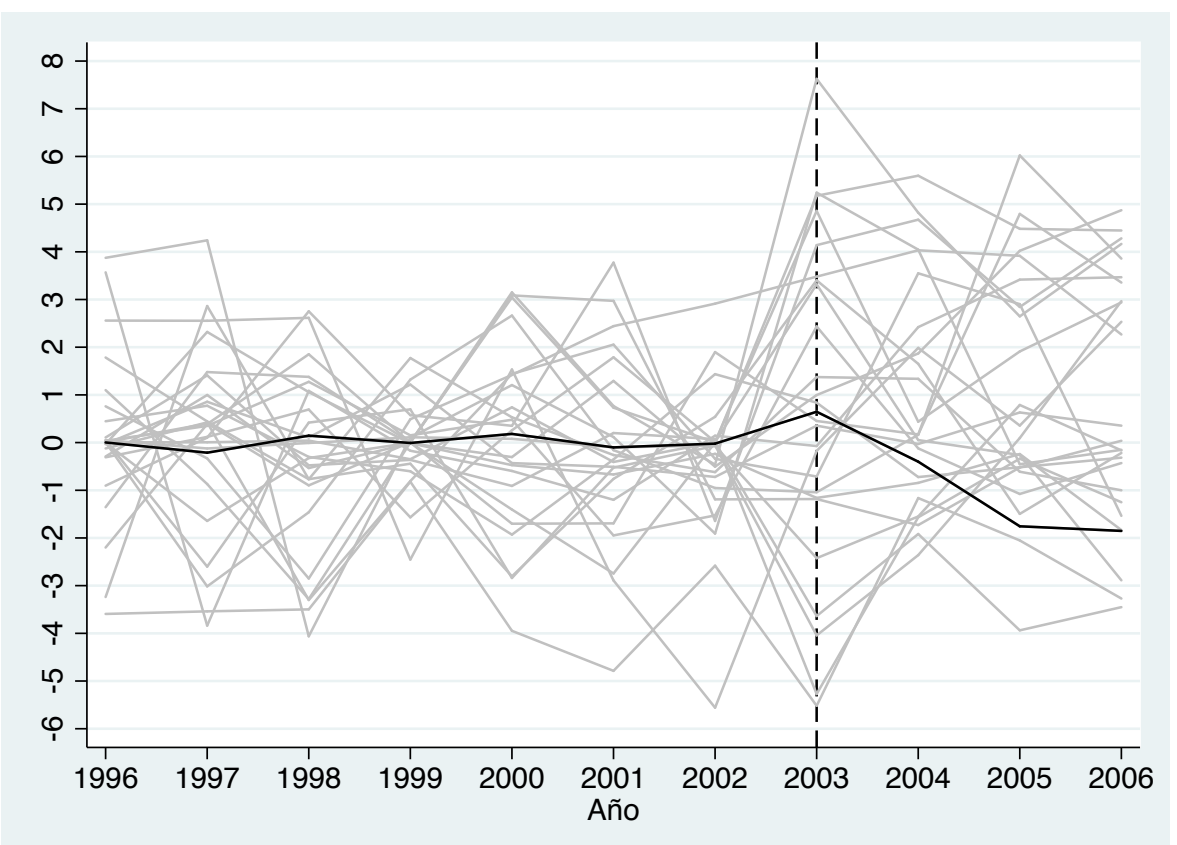

Figura 5: Brecha en el porcentaje de individuos que habitan viviendas inadecuadas: San Luis y 25 aglomerados placebo.

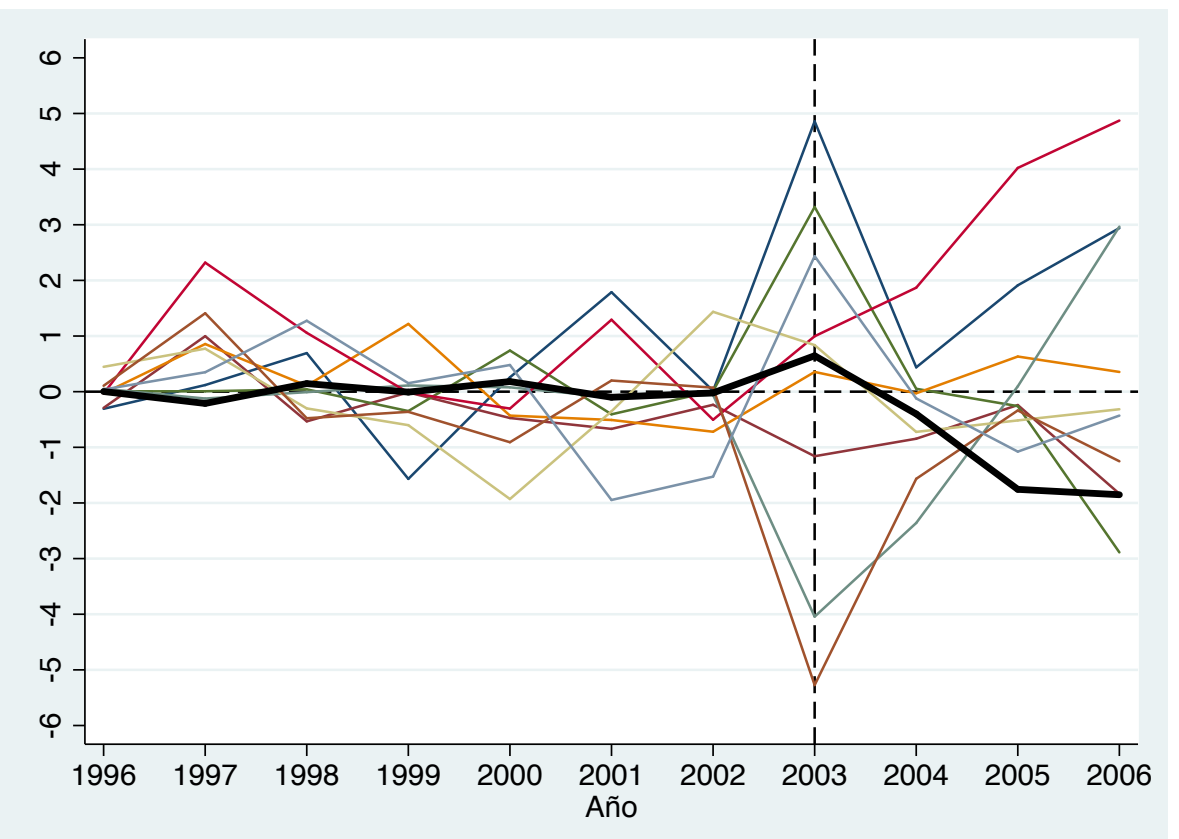

Figura 6: Brecha en el porcentaje de individuos que habitan viviendas inadecuadas: San Luis y 9 aglomerados placebo. 
(a) Sin agua (\%)

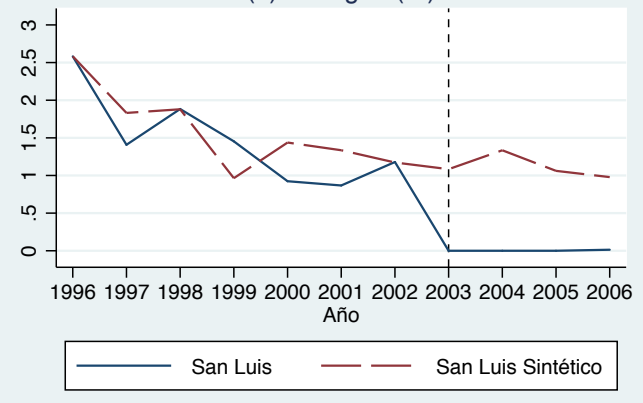

(c) Con hacinamiento (\%)

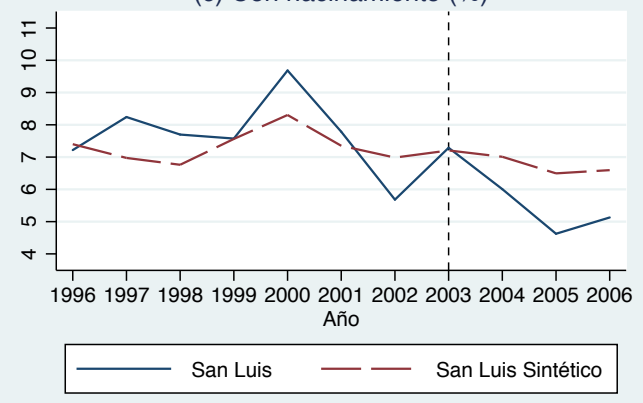

(b) Con materiales deficientes (\%)

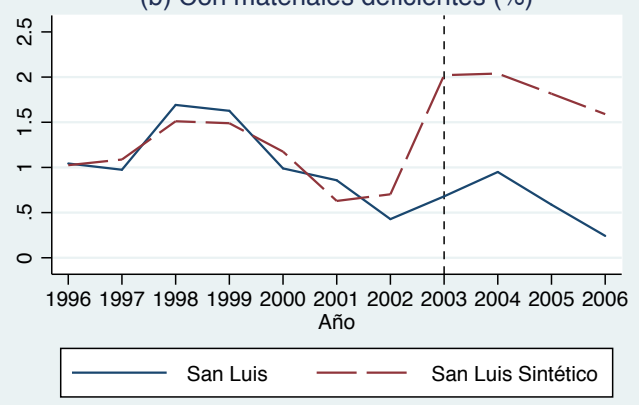

(d) Sin baño (\%)

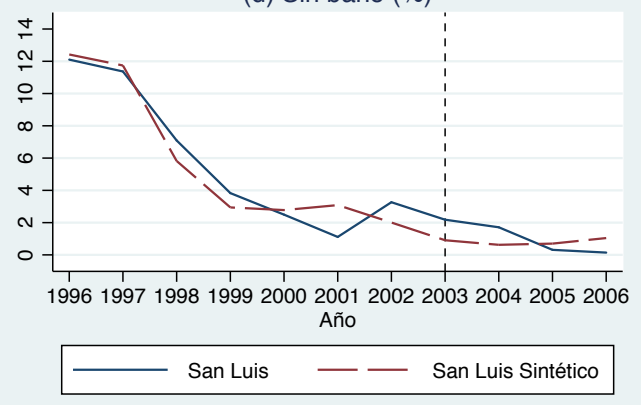

Figura 7: Tendencias en el porcentaje de individuos que habitan viviendas (a) Sin conexión a agua corriente, (b) Con materiales deficientes, (c) Con hacinamiento y (d) Sin baño de uso exclusivo: San Luis Real y Sintético.

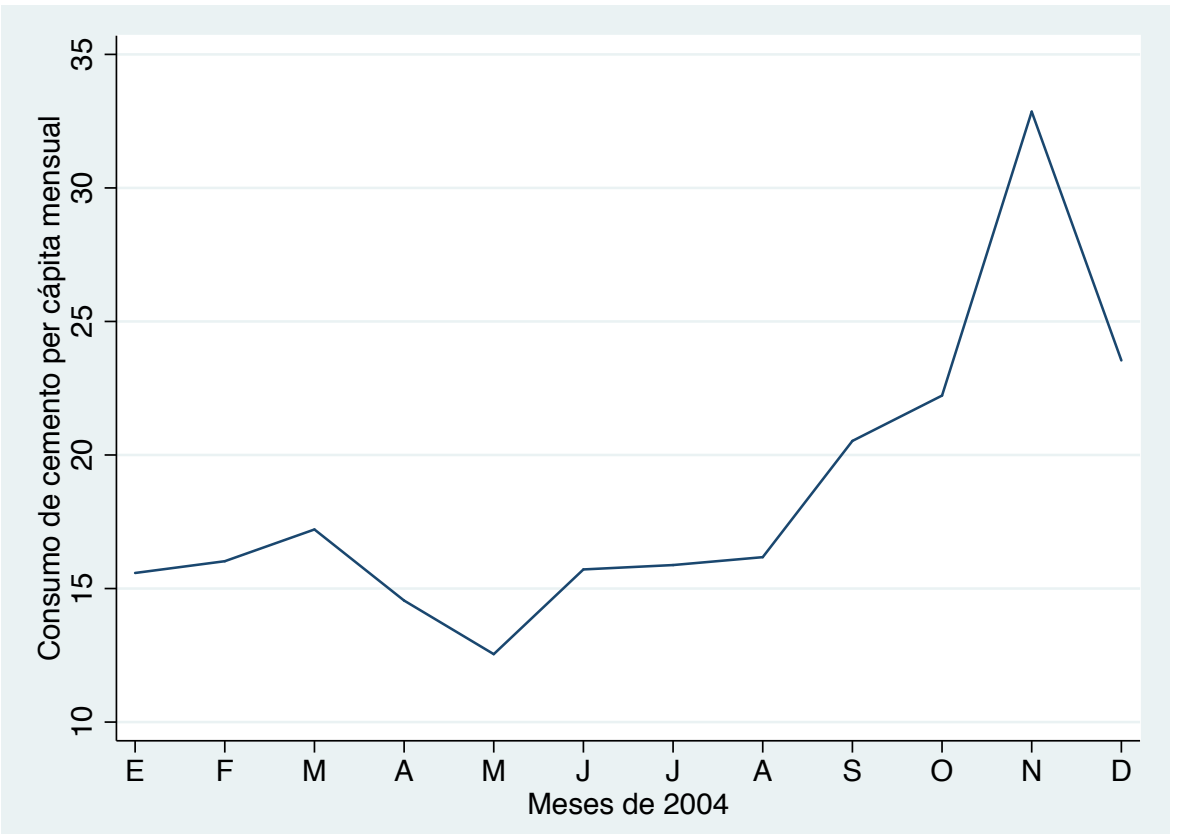

Figura 8: Evolución del consumo de cemento per cápita mensual en San Luis durante 2004. 
Tabla 3: Estimaciones del modelo econométrico de consumo de cemento per cápita a nivel mensual. Año 2004.

\begin{tabular}{|c|c|c|}
\hline Variables & $\mathrm{EF}$ & $\mathrm{EA}$ \\
\hline Dummy San Luis (=1 Si) & - & $\begin{array}{c}2.073 \\
(1.646)\end{array}$ \\
\hline Febrero*San Luis & $\begin{array}{l}1.861 \\
(1.376)\end{array}$ & $\begin{array}{c}1.861 \\
(1.379)\end{array}$ \\
\hline Marzo*San Luis & $\begin{array}{l}1.143 \\
(1.159)\end{array}$ & $\begin{array}{c}1.143 \\
(1.161)\end{array}$ \\
\hline Abril*San Luis & $\begin{array}{c}0.357 \\
(0.914)\end{array}$ & $\begin{array}{c}0.357 \\
(0.915)\end{array}$ \\
\hline Mayo*San Luis & $\begin{array}{c}-1.613^{* *} \\
(0.754)\end{array}$ & $\begin{array}{c}-1.613^{* *} \\
(0.755)\end{array}$ \\
\hline Junio*San Luis & $\begin{array}{l}1.710 \\
(1.299)\end{array}$ & $\begin{array}{c}1.710 \\
(1.302)\end{array}$ \\
\hline Julio*San Luis & $\begin{array}{l}1.797 \\
(1.522)\end{array}$ & $\begin{array}{l}1.797 \\
(1.525)\end{array}$ \\
\hline Agosto*San Luis & $\begin{array}{c}0.659 \\
(1.279)\end{array}$ & $\begin{array}{c}0.659 \\
(1.281)\end{array}$ \\
\hline Septiembre*San Luis & $\begin{array}{c}3.563^{* * *} \\
(1.218)\end{array}$ & $\begin{array}{c}3.563^{* * *} \\
(1.220)\end{array}$ \\
\hline Octubre*San Luis & $\begin{array}{c}3.677^{* * *} \\
(0.599)\end{array}$ & $\begin{array}{c}3.677^{* * *} \\
(0.600)\end{array}$ \\
\hline Noviembre*San Luis & $\begin{array}{c}15.072^{* * *} \\
(1.297)\end{array}$ & $\begin{array}{c}15.072^{* * *} \\
(1.299)\end{array}$ \\
\hline Diciembre*San Luis & $\begin{array}{c}6.038^{* * *} \\
(0.869)\end{array}$ & $\begin{array}{c}6.038^{* * *} \\
(0.870)\end{array}$ \\
\hline Constante & $\begin{array}{l}13.595 \\
(0.837) \\
\end{array}$ & $\begin{array}{l}13.508 \\
(1.646) \\
\end{array}$ \\
\hline EF x Mes & $\mathrm{Si}$ & $\mathrm{Si}$ \\
\hline EF x Provincia & $\mathrm{Si}$ & No \\
\hline $\mathrm{R} 2$ & 0.288 & 0.288 \\
\hline Obs. & 288 & 288 \\
\hline
\end{tabular}

Notas: Errores estándar robustos entre paréntesis.

Significancia estadística al 1\%, $5 \%$ y $10 \%$ denotada por $* * *, * * \mathrm{y}^{*}$, respectivamente. 